\title{
Concentration and composition dependent aggregation of Pluronic- and Poly-(2-oxazolin)-Efavirenz formulations in biorelevant media
}

\author{
Sebastian Endres a , Emil Karaev ${ }^{\mathrm{a}}$, Simon Hanio ${ }^{\mathrm{b}}$, Jonas Schlauersbach ${ }^{\mathrm{b}}$, Christian Kraft ${ }^{\mathrm{c}}$, \\ Tim Rasmussen ${ }^{\mathrm{c}, \mathrm{d}}$, Robert Luxenhofer ${ }^{\mathrm{e}}$, Bettina Böttcher ${ }^{\mathrm{c}, \mathrm{d}}$, Lorenz Meinel ${ }^{\mathrm{b}, \mathrm{f}}$, Ann-Christin Pöppler ${ }^{\mathrm{a}, *}$ \\ a Institute of Organic Chemistry, University of Würzburg, Am Hubland, Würzburg 97074, Germany \\ ${ }^{\mathrm{b}}$ Institute of Pharmacy and Food Chemistry, University of Würzburg, Am Hubland, Würzburg 97074, Germany \\ ${ }^{\mathrm{c}}$ Rudolf Virchow Center, Center for Integrative and Translational Bioimaging, University of Würzburg, Würzburg 97080, Germany \\ ${ }^{\mathrm{d}}$ Biocenter, University of Würzburg, Würzburg 97074, Germany \\ e Soft Matter Chemistry, Department of Chemistry, Helsinki University, Helsinki 00014, Finland \\ ${ }^{\mathrm{f}}$ Helmholtz Institute for RNA-based Infection Biology (HIRI), Wuerzburg DE-97070, Germany
}

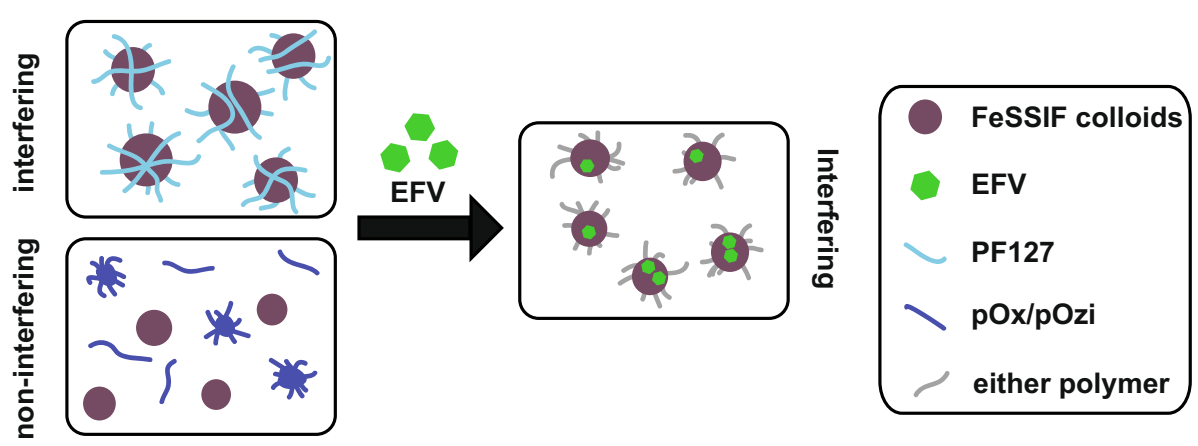

\section{A R T I C L E I N F O}

\section{Article history:}

Received 29 May 2021

Revised 26 July 2021

Accepted 7 August 2021

Available online 10 August 2021

\section{Keywords:}

Bile colloids

Drug-Polymer Formulations

Concentation dependent interaction

DOSY

\begin{abstract}
A B S T R A C T
Many drugs and drug candidates are poorly water-soluble. Intestinal fluids play an important role in their solubilization. However, the interactions of intestinal fluids with polymer excipients, drugs and their formulations are not fully understood. Here, diffusion ordered spectroscopy (DOSY) and nuclear Overhauser effect spectroscopy (NOESY), complemented by cryo-TEM were employed to address this. Efavirenz (EFV) as model drug, the triblock copolymers Pluronic ${ }^{\circledR}$ F-127 (PF127) and poly(2-oxazoline) based pMeOx- $b$ pPrOzi- $b$-pMeOx (pOx/pOzi) and their respective formulations were studied in simulated fed-state intestinal fluid (FeSSIF). For the individual polymers, the bile interfering nature of PF127 was confirmed and pure pOx/pOzi was newly classified as non-interfering. A different and more complex behaviour was however observed if EFV was involved. PF127/EFV formulations in FeSSIF showed concentration dependent aggregation with separate colloids at low formulation concentrations, a merging of individual particles at the solubility limit of EFV in FeSSIF and joint aggregates above this concentration. In the case of pOx/pOzi/EFV formulations, coincident diffusion coefficients for pOx/pOzi, lipids and EFV indicate joint aggregates across the studied concentration range. This demonstrates that separate evaluation of polymers and drugs in biorelevant media is not sufficient and their mixtures need to be studied to learn about concentration and composition dependent behaviour.

(c) 2021 The Authors. Published by Elsevier Inc. This is an open access article under the CC BY license (http://
\end{abstract} creativecommons.org/licenses/by/4.0/).

\footnotetext{
* Corresponding author.

E-mail address: ann-christin.poeppler@uni-wuerzburg.de (A.-C. Pöppler).
} 


\section{Introduction}

Approximately $40 \%$ of worldwide approved drugs are poorly water-soluble and categorized as class II and class IV in the Biopharmaceutics Classification system (BCS).[1,2] The even higher amount in drug candidates has increased from $60 \%$ reported in 2013 [1] to $70 \%$ - 90\% in 2017 [2]. In this context, drug delivery systems (DDS) such as nano- or microparticles based on lipids and polymers are investigated to overcome solubility challenges or generally alter physicochemical properties and influence pharmacokinetics.[3] Since the mid-1990 s, the number of publications on this topic strongly increased (Figure S1). Recent reports stressed, however, that the large number of scientific publications on drug delivery is not mirrored by corresponding therapeutic advances.[4] An academic focus on very complex systems compromising manufacturability and reproducibility was discussed as a potential problem source.[4-6] Polymers responding to various self- and externally regulated stimuli e.g. $\mathrm{pH},[7,8]$ redox potentials,[7,9] urea concentration [7] or light $[10,11]$ and drug conjugated polymers[12,13] are investigated. $>85 \%$ of formulations entering clinical trials fail at this stage.[14,15] Most of these failures are associated with efficacy or safety problems, which shows that many compounds and formulations do not have the clinical effect they were designed for. An optimized selection protocol preceding admission to clinical trials could increase their success rate and be more time and cost efficient. Such an optimized selection protocol could address apparent differences between in vitro and in vivo behaviour and consider the multifunctional nature of some nanoformulations.[14,16]

For orally administered drugs a mechanistical understanding of processes in the small intestine, the major site for drug absorption, is essential for drug development.[17] After being swallowed, the drugs encounters an acidic environment with digestive enzymes in the stomach, before entering the small intestine.[18,19] The intestinal fluids and their composition play an important role in solubilisation of drugs[20-22] and their subsequent absorption. $[16,23]$ Therefore, simulated biorelevant media such as FaSSIF (Fasted State Simulated Intestinal Fluid) or FeSSIF (Fed State Simulated Intestinal Fluid) were developed to study the influence of bile on physicochemical parameters in vitro.[24-35] Key components in these simulating media are sodium taurocholate representing bile and lecithin. While simulated media are naturally a simplified version of their in vivo counterpart, detailed morphological investigations of human intestinal fluids and FaSSIF/FeSSIF mixtures indicated a comparable particle composition.[36] In practice, significant changes in drug solubility between aqueous buffer solutions and biorelevant media suggest re-assignment of various drugs to a different BCS class.[37]

Moreover, an increasing number of reports also shows the interaction of bile colloids with different polymer excipients, e.g.
Pluronics ${ }^{\circledR},[38,39]$ Triton $^{\circledR}$ X-100,[40] Eudragit $^{\circledR}$ E or Soluplus ${ }^{\circledR}$ [41] and thus their influence on the mutual aggregation behaviour. Consequently, the inclusion of polymeric excipients, solubility enhancers and transport vehicles into formulations adds a layer of complexity as the additional and largely unexplored polymerdrug and polymer-bile interactions affect the existing drug-bile interactions.[42] Other studies could show the effect of drug concentration on the colloidal aggregates within the intestinal fluids. [43]This underlines the need for thorough investigations of complex mixtures of DDS in biorelevant media to gain a better understanding and an improved predictability of the in vivo behaviour. [44] Previous studies relied on flux measurements of polymeric drug formulations across artificial membranes in combination with differential scanning calorimetry (DSC).[45] ${ }^{1} \mathrm{H}$ - and DOSY (diffusion ordered spectroscopy) NMR spectroscopy were also identified as suitable tools in the investigation of drug loaded surfactant colloids in FaSSIF $[28,46]$ or lipid free bile salt solutions.[47,48] Both of these NMR spectroscopic studies focus on either the drug molecule or the polymeric carrier material, while it is to be expected that the individual drug can also change the behaviour of the polymer in biorelevant media and vice versa. Furthermore, there are new and experimental polymer platforms, whose behaviour in biorelevant media is yet unexplored.

To understand the multifaceted and dynamic nature of the aggregates present in mixtures of biorelevant media and polymer-drug formulations in detail, similarly diverse analytical tools are required. In this project, this was realised through an NMR spectroscopic toolbox focussing on DOSY for the analysis of particle sizes and (hetero)nuclear Overhauser spectroscopy (NOESY, selective NOESY, HOESY) for the investigation of spatial proximities. These data were complemented by cryotransmission electron microscopy (cryo-TEM) measurements. FeSSIF-V2 (hereinafter referred to as FeSSIF) as recommended by the United States Pharmacopeia (USP) for solubility measurements was chosen as biorelevant medium to reflect the fed state.

Efavirenz (EFV, Fig. 1), a BCS class II compound (estimated aqueous solubility of $11.5 \frac{\mathrm{mg}}{\mathrm{L}}, 36 \mu \mathrm{M}$ ) [49,50]employed in the treatment of HIV-1 infections, was selected as model compound.[51] Its increased solubility in FeSSIF ( $880 \frac{\mathrm{mg}}{\mathrm{L}}, 2.8 \mathrm{mM}$ at $37^{\circ} \mathrm{C}$ ) serves as a basis for this investigation.[52] EFV solubility can also be increased by incorporation into polymeric micelles such as Pluronic $^{\circledR}$ F127 (PF127), an FDA approved and commercially available polymer (Fig. 1).[53,54] PF127 is a well-studied thermoresponsive polymer comprising of two polyethylene oxide (PEO) blocks (mean block length $\sim 100$ units) with a polypropylene oxide (PPO) central block (mean block length $\sim 56$ units).[55] Alternatively, polymers based on poly(2-oxazolines) are an emerging platform for biomedical applications [56,57] showing advantageous properties in the formulation of hydrophobic drugs and structural modularity.[56-59] They previously showed to efficiently formu-<smiles>[CH]OCC(C)(C)OC(C)CC(C)(C)OCC[C@H](C)O</smiles>

Pluronic ${ }^{\circledR}$ F-127 (PF127)

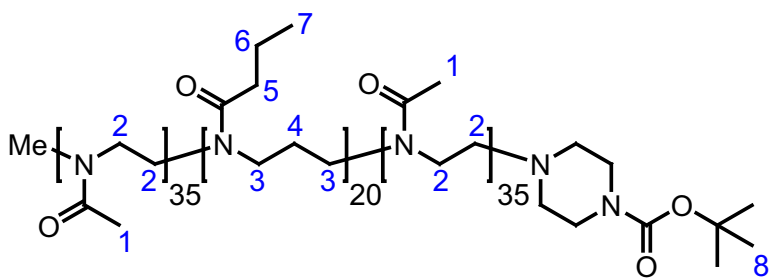

pMeOx-b-pPrOzi-b-pMeOx (pOx/pOzi)

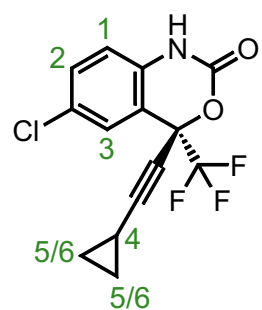

Efavirenz (EFV)

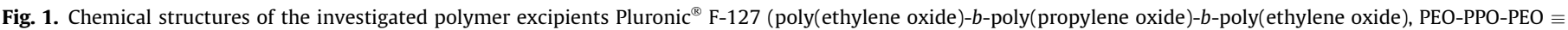

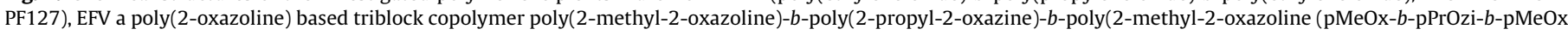
$\equiv \mathrm{pOx} / \mathrm{pOzi})$ and $\mathrm{EFV}$ including numbering of relevant atom positions. 
late EFV.[60] Therefore, an A-B-A tri-block copolymer consisting of hydrophilic poly(2-methyl-2-oxazoline) blocks (A, 35 units) and a more hydrophobic poly(2-propyl-2-oxazine) (B, 20 units) block (pOx/pOzi) was studied (Fig. 1). While previous reports supported the classification of PF127 as bile salt interfering polymer,[61,62] to date no data on the behaviour of poly(2-oxazolines) in biorelevant media has been reported.

\section{Materials and methods}

\subsection{Materials}

FeSSIF-V2 powder was purchased from Biorelevant.com (UK). Deuterium oxide, DMSO $d_{6}, \mathrm{NaOD}$ and $\mathrm{DCl}$ were purchased form Deutero GmbH (Germany) with a purity of $99.9 \%$. EFV was purchased (>98\% purity) from TCI Chemicals (Germany), PF-127, suitable for cell culture, from Merck (Germany). pOx/pOzi and the corresponding EFV formulation were prepared according to literature.[60] All other standard chemicals and laboratory consumables were purchased from either VWR International GmbH (Germany) or Merck (Germany).

\subsection{Sample preparation}

FeSSIF-V2 solution was prepared, according to the protocol of Biorelevant. Instead of protonated water, deuterated water was used and the apparent $\mathrm{pH}_{\mathrm{a}}=6.26$ was adjusted from 5.8 to 6.26 using NaOD and $\mathrm{DCl}$ to account for a different apparent $\mathrm{pH}$ due to the isotope effect.[63] The exact composition is shown in Table S2. Solutions of EFV in FeSSIF were prepared by the solvent switch method adding $5 \mu \mathrm{L}$ of an EFV stock solution in DMSO $d_{6}$ to $500 \mu \mathrm{L}$ FeSSIF solution and shaken for 2 min to achieve concentrations of 1 to $10 \mathrm{mM}$ EFV. This concentration range resembles the expected concentrations after the dissolution of a $200 \mathrm{mg}$ EFV dose [64] in a volume between $18 \mathrm{~mL}(\sim 37 \mathrm{mM})$ and $660 \mathrm{~mL}(\sim 1 \mathrm{mM})$, the recorded volume of intestinal fluid in the fed state.[65-67] Solutions of polymers and their EFV formulations were prepared by dissolution in FeSSIF.

\subsection{NMR spectroscopy}

All NMR spectra were recorded at a Bruker Avance III HD spectrometer (Germany) operating at $14.1 \mathrm{~T}$ using either a $5 \mathrm{~mm}$ BBFO probe or a $5 \mathrm{~mm} \mathrm{DCH}$ cryo-probe, both equipped with z-gradient and a temperature control unit, unless stated otherwise. Trimethylsilylpropanoic acid- $\mathrm{d}_{4}$ in $\mathrm{D}_{2} \mathrm{O}$ was used in coaxial inserts tubes as an internal chemical shift reference and all experiments were recorded at $37{ }^{\circ} \mathrm{C}$ to resemble physiological conditions. Before and after longer experiments (e.g. NOESY or HSQC) a ${ }^{1} \mathrm{H}$ NMR spectrum was acquired to check for apparent changes during the measurement. Data were analysed using the TopSpin 3.6.0 software.

\subsection{1. $2 D^{1}{ }^{H}-{ }^{1} \mathrm{H} N O E S Y$}

Two dimensional ${ }^{1} \mathrm{H}-{ }^{1} \mathrm{H}$ NOESY spectra were recorded using the noesygpphpp sequence with a recycle delay of $>3.5$ s. For selected samples, NOESY was recorded with three different mixing times ( $\mathrm{d} 8$ ) of $20 \mathrm{~ms}, 40 \mathrm{~ms}$ and $60 \mathrm{~ms}$ to check whether the intensity of off-diagonal resonances increases linearly. All measurements were conducted with a mixing time of $60 \mathrm{~ms}$ or lower, unless signal enhancement by spin diffusion was intended.

\subsubsection{Selective NOE experiments}

Selective ${ }^{1} \mathrm{H}-{ }^{1} \mathrm{H}$ NOESY spectra were acquired using the selnogp pulse sequence with a recycle delay of $4.48 \mathrm{~s}$. For the selective inversion either a "Rsnob.1000" or a "Gaus1_180r.1000" with pulse lengths and powers adjusted for optimal excitation were used and tested using the selgpse pulse sequence.

\subsubsection{Dosy NMR}

Pulsed-field-gradient (PFG) NMR spectra were acquired using the ledbpgp2s [68] or the dstebpgp3s [69,70] pulse sequences without rotation. To avoid convection effects due to temperature gradients within the probe only the double stimulated echo sequence was used when measuring with the DCH cryo-probe with a maximum gradient strength of $57 \mathrm{G} \mathrm{cm}^{-1}$. The BBFO probe, with a maximum gradient strength of $50 \mathrm{G} \mathrm{cm}^{-1}$, does not exhibit a large temperature gradient. As no differences were observed between the sequences with and without convection compensation for the BBFO probe, spectra on this probe were recorded with the ledpbgp2 pulse sequence. For all experiments, the diffusion gradients were linearly incremented in 32 steps from 2 to $98 \%$. The diffusion time (d20) was set to $100 \mathrm{~ms}$ and the length of the gradient pulses ( $\mathrm{p} 30$ ) was adjusted to achieve a signal attenuation of below $1 \%$.

The obtained attenuation curves were fitted using one of the following options: (i) If possible, a mono-exponential fit was used. In cases of a poor match either a (ii) bi-exponential fit, or (iii) a fit assuming a log-normal distribution were employed. The lognormal distributions were adapted from the work of Guo et al. [71] A detailed description of the fitting process is given in the SI. All fitting procedures were performed using Origin 2020 software (OriginLab ${ }^{\circledR}$ ). Mean values and deviations for the DOSY summaries were calculated using all unambiguous data points corresponding to the same molecules within one diffusion experiment. Error bars shown in DOSY plots then indicate the standard deviations of averaged data points.

Hydrodynamic diameters from PFG experiments were estimated using the Stokes-Einstein equation assuming spherical aggregates and a viscosity of $0.7866 \mathrm{mPa} \cdot \mathrm{s}$ (viscosity of deuterated water at $40^{\circ} \mathrm{C}[72]$ ) to facilitate the comparison between DOSY and TEM data.

\subsection{CrYoTEM}

$3.5 \mu \mathrm{L}$ of sample was applied to glow-discharged, holey carbon coated copper 400 grids 1.2/1.3(Quantifoil) and vitrified in liquid ethane with a Vitrobot mark IV (FEI) with $3 \mathrm{~s}$ blot time and $100 \%$ humidity at $37{ }^{\circ} \mathrm{C}$. Micrographs were collected on a Titan Krios G3 (Thermo Scientific) operated at $300 \mathrm{kV}$. The magnification was set to 75,000 (calibrated pixel size $1.0635 \AA$ A/pixel) and images were recorded with a total exposure of $80 \mathrm{e}^{-} / \AA^{2}$ and a nominal defocus of $2.5 \mu \mathrm{m}$ on a Falcon III direct detector in integrating mode. All pictures were processed and analysed using ImageJ. The script for data processing is shown in the SI.

\section{Results and discussion}

\section{1. ${ }^{1} \mathrm{H}$ NMR signal assignment}

FeSSIF is a mixture of sodium taurocholate (TC) and poorly water soluble lipids lecithin (LC), sodium oleate and glycerol monooleate (fatty acids $\equiv \mathrm{FA}$ ) in a saline maleic acid buffer at $\mathrm{pH}$ 6.26 forming mixed aggregates (Fig. 2).[73] In order to understand and keep track of changes on the molecular level or investigate through-space interactions via NMR spectroscopy a complete resonance assignment was necessary. Despite substantial signal overlap, broadening and higher order splittings a complete assignment based on different 2D correlations was possible. 
A:<smiles>C[N+]([O-])(I)CCOP(=O)(O)OC([12CH3])[12CH3]</smiles>

B:<smiles>[14CH3]C(O)C(O)O</smiles>

c: $\overbrace{2}^{5} \int_{5}^{\mathrm{OH}} \mathrm{OH}$

R: 1

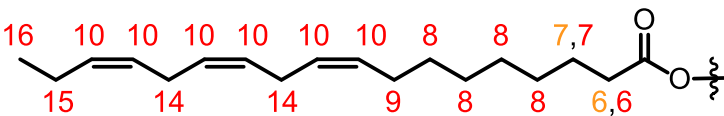

$\mathbf{R}^{\prime}$ :

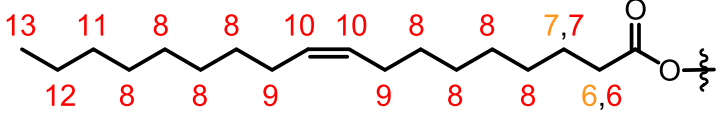

$A+R / R^{\prime}=$ Lecithin (LC)

$\mathrm{B} / \mathrm{C}+\mathrm{R}=$ Glycerol Monooleate (FA)

$\mathbf{R}=$ Oleate (FA)

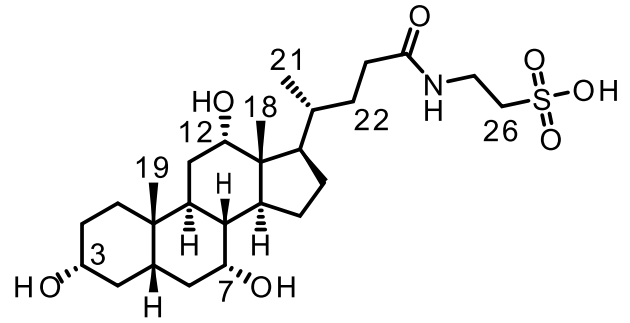

Taurocholic Acid (TC)

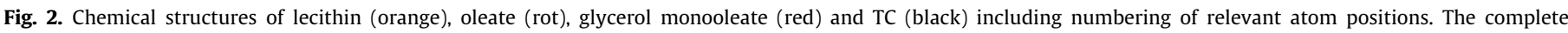
numbering for TC is shown in the SI (Figure S6).

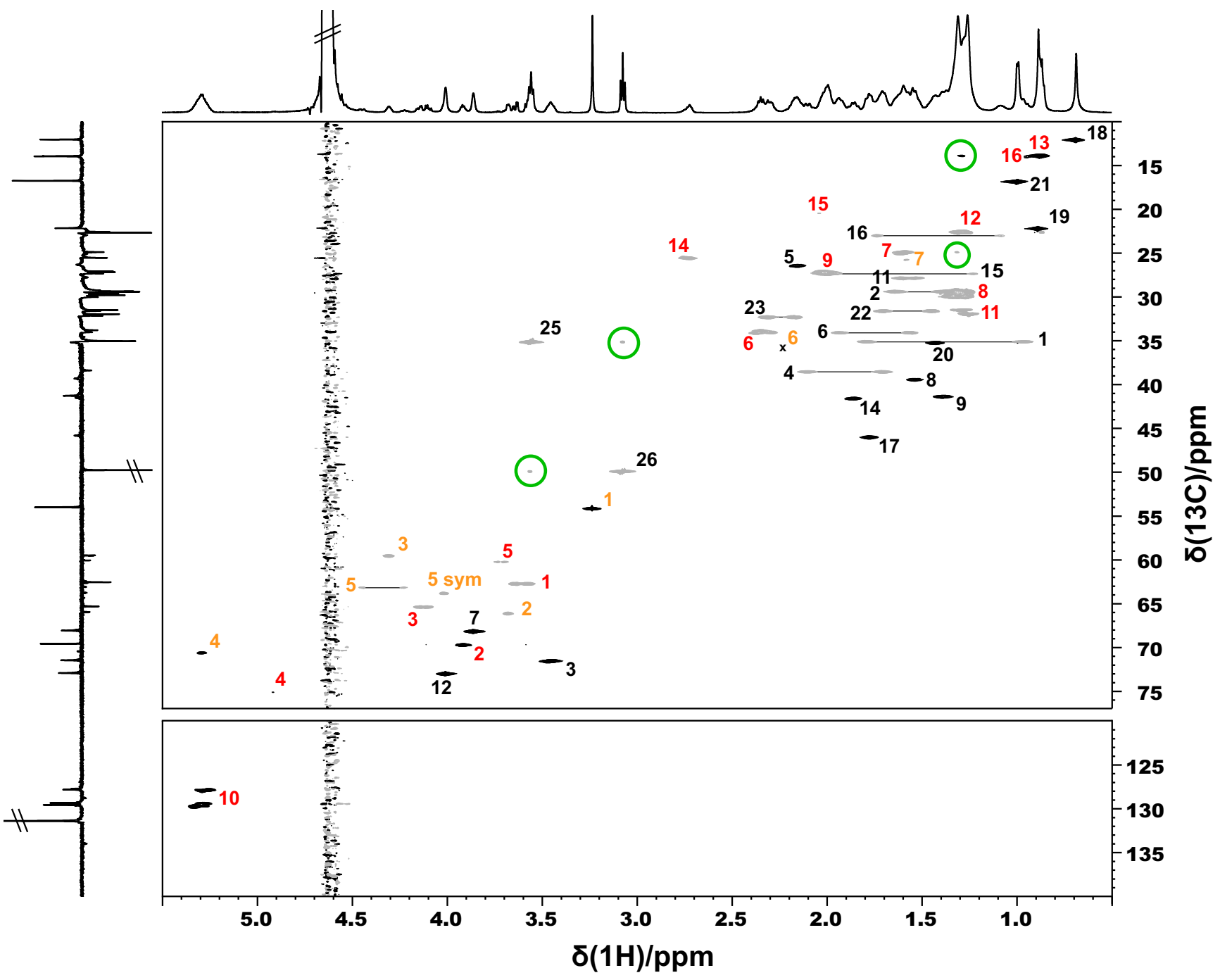

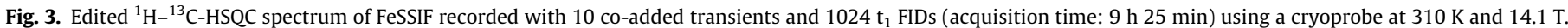

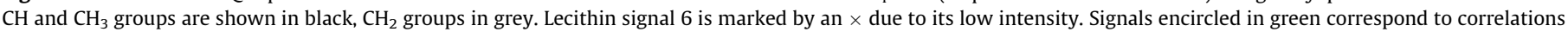

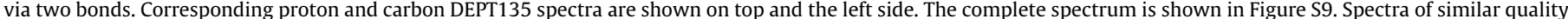

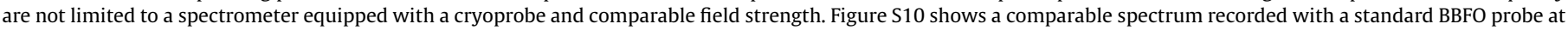
$9.4 \mathrm{~T}$. 
Fig. 3 shows the ${ }^{1} \mathrm{H}-{ }^{13} \mathrm{C}-\mathrm{HSQC}$ correlation of FeSSIF including the complete assignment of the detectable peaks. Starting from the characteristic methyl peaks between 0.7 and $1 \mathrm{ppm}$, the surrounding TC protons were assigned using COSY and HMBC spectra. Due to the low concentration of the sample not all anticipated cross-correlation signals could be detected. Ambiguous assignments were clarified using ${ }^{1} \mathrm{H}-{ }^{1} \mathrm{H}-\mathrm{NOESY}$ data (Figure S15). Fatty acids, glycerides and lecithin were assigned starting from the vinyl protons and HMBC correlations to the carbonyl carbons.

\subsection{Analysis of ${ }^{1} \mathrm{H}$ chemical shift changes}

To study the behaviour of polymer drug formulations in biorelevant media in detail on a molecular level, different mixtures of FeSSIF were prepared. FeSSIF/EFV samples were prepared using the solvent switch method with concentrations ranging from $1 \mathrm{mM}$ to $10 \mathrm{mM}$ to resemble the uptake of a $200 \mathrm{mg}$ EFV dose in the fed state small intestine with recorded volumes between 18 and $660 \mathrm{~mL}$ [65-67] However, at concentrations of $6 \mathrm{mM}$ or higher, the solutions were supersaturated and formed murky gels within $1 \mathrm{~h}$. After several hours, crystallized EFV was also observed in the $5 \mathrm{mM}$ sample, while the remaining samples showed no signs of gelation or crystallization within three consecutive days. These dissolution experiments in FeSSIF resulted in an estimated EFV solubility enhancement by a factor of about 135 compared to pure water. This could contribute to a generally high bioavailability of EFV [51] despite its poor aqueous solubility.[50] All other samples were prepared by dissolution of the polymers $(0.1 \%, 0.5 \%$ and $1 \%$ by weight) or their corresponding freeze-dried EFV formulations $(0.1 \%, 0.2 \%, 0.3 \%, 0.4 \%, 0.5 \%$ and $1 \%$ polymer by weight) in FeSSIF.

Addition of drugs, polymers or formulations can lead to changes in the size, composition and structure of bile colloids, which are expected to impact the proton spectra of these mixtures. Such changes in the microenvironment can be a first and easily accessible indicator for altered aggregation phenomena. For an overview of these influences, changes in chemical shift relative to pure FeSSIF were measured for signals that showed an unambiguous peak centre and no severe signal overlap (Fig. 4). The top three graphs (Fig. 4 a, b, c) focus on PF127 and its EFV formulations in FeSSIF, the lower row contains the corresponding data for pOx/pOzi (Fig. 4 a, d, e). For the solutes EFV and PF127 the respective spectrum with the lowest concentration was used as reference. pOx/ pOzi resonances were superimposed in most spectra, therefore hampering the extraction of unambiguous data points. The observed chemical shift changes were in the range of -10 to $+35 \mathrm{~Hz}$ and thus comparably small, indicative of a dynamic system.

The increased solubility of pure EFV in FeSSIF solutions already indicated an incorporation of EFV into bile aggregates. This is further supported by changes in EFV chemical shifts resulting from different micro-environments (Fig. 4a): FeSSIF samples with varying amounts of pure EFV show the largest chemical shift difference with up to $-30 \mathrm{~Hz}$. Here, EFV and TC signals shift uniformly to smaller values, while no clear trend can be observed for LC/FA signals. For PF127, an increasing polymer concentration in FeSSIF samples (Fig. 4b) affected the chemical shifts moderately with the non-polar fatty acids (red symbols) being influenced strongest $(+12 \mathrm{~Hz}$ at $1 \mathrm{wt} \%$ polymer concentration). Comparison of Fig. 4a (FeSSIF/EFV) and Fig. 4c (FeSSIF/PF127/EFV) shows larger chemical shift changes for samples with EFV in the absence of PF127, suggesting mixed or co-existing aggregates when both EFV and PF127 are present. Interestingly, chemical shift changes of aromatic proton EFV 1 (green squares) are opposite: $\Delta \delta=-10 \mathrm{~Hz}$ and $+11 \mathrm{~Hz}$ for FeSSIF/EFV and FeSSIF/PF127/EFV, respectively.

In contrast, for different concentrations of pOx/pOzi in FeSSIF only very small ${ }^{1} \mathrm{H}$ chemical shift changes $(<5 \mathrm{~Hz})$ were observed
(Fig. 4d). Therefore, on the molecular level, FeSSIF is mostly unaffected by the presence of $\mathrm{pOx} / \mathrm{pOzi}$. For the $\mathrm{pOx} / \mathrm{pOzi} / \mathrm{EFV}$ formulation in FeSSIF, the situation is more complex (Fig. 4e): (i) For pOx/ pOzi/EFV formulations in $\mathrm{D}_{2} \mathrm{O}$ only one broad signal with a linewidth $>300 \mathrm{~Hz}$ (Figure S11) was visible in the ${ }^{1} \mathrm{H}$ NMR spectra and for pure EFV in FeSSIF linewidths of the EFV signals were $<40 \mathrm{~Hz}$. Interestingly, signals with a line width $<40 \mathrm{~Hz}$ were observed for the pOx/pOzi/EFV formulation in FeSSIF. (ii) While the presence of the polymer alone did not have a significant influence on the chemical shifts of the FeSSIF components, the addition of EFV to this mixture led to stronger changes in chemical shift. (iii) The largest changes were observed for EFV $(-36 \mathrm{~Hz}$ at $0.5 \mathrm{wt} \%$ polymer) and TC ( $-26 \mathrm{~Hz}$ at $0.5 \mathrm{wt} \%$ polymer). These values are comparable to mixtures without polymer at comparable EFV concentrations (Fig. 4c). However, the overall higher solubility of EFV in FeSSIF in the presence of pOx/pOzi suggests that FeSSIF can interact with pOx/pOzi micelles to exchange EFV.

Based on the chemical shift data it can be assumed that the polymers interact with FeSSIF in different ways. Especially in combination with a small-molecule drug, all components exhibit different chemical environments. ${ }^{1} \mathrm{H}-{ }^{1} \mathrm{H}-\mathrm{NOESY}$ and ${ }^{1} \mathrm{H}$-DOSY NMR complemented by cryo-TEM are employed for a more detailed investigation of the nature of the aggregates formed in these multicomponent mixtures.

\subsection{EFV in FeSSIF}

Apart from chemical shift differences, peak shapes and widths are also indicative of changes on the molecular level. An overall broadening of the signals was observed with increasing EFV concentration (Figure S12). Such broadening can be either due to reduced mobility or a distribution of differing chemical environments. Interestingly, in a ${ }^{1} \mathrm{H}^{-13} \mathrm{C}$ HSQC spectrum, aromatic $\mathrm{C}-\mathrm{H}$ correlations involving EFV could not be detected even after longer measurement time and using various optimized $\mathrm{C}-\mathrm{H}$ coupling constants or different pulse sequences. Furthermore, TC signals were also much weaker than in pure FeSSIF. Combining these three observations suggests that in EFV/FeSSIF mixtures, EFV either interacts with TC or with the lipids, which in turn results in a different TC environment and an increased EFV solubility. To investigate these hypotheses based on observable through-space interactions ${ }^{1} \mathrm{H}-{ }^{1} \mathrm{H}$ NOESY spectra with a short mixing time of $50 \mathrm{~ms}$ (Figure S16) were recorded. Strong absorptive interactions between the aromatic protons 1 and 2 of EFV, as well as weaker peaks between the aromatic protons and the three-membered ring could be detected. Additionally, a weak contact between the aromatic resonance and FA 8/11/12 was identified. Due to the lack of NOESY resonances between EFV and the remaining components, the mixing time was increased to $0.5 \mathrm{~s}$ and $1 \mathrm{~s}$. This resulted in more cross-peaks between EFV and lipids, FA 10/9/7 (hydrophobic) and LC 1 (polar) and weak contacts to TC 25/26 could be identified. Interactions between TC and lipids, which were present in pure FeSSIF at a mixing time of $0.4 \mathrm{~s}$, were observed with strongly reduced intensity. Since EFV is nearly insoluble in water it seems to be predominantly solubilised by the lipids resulting in spatial proximity and therefore NOESY contacts between both substances, but also between EFV itself. This changes the environment of TC, which was initially incorporated more strongly in the particles and is now edged out by hydrophobic EFV, which would result in reduced contacts to lipids. The EFV-amide moiety could furthermore interact weakly with more polar functionalities such as the corresponding amide in TC.

Pulsed field gradient (PFG) NMR is a powerful tool to study aggregation as similar diffusion coefficients indicate interdependent diffusion and therefore mixed aggregates. In some cases, molecules co-exist in aggregates and in solution. Depending on 


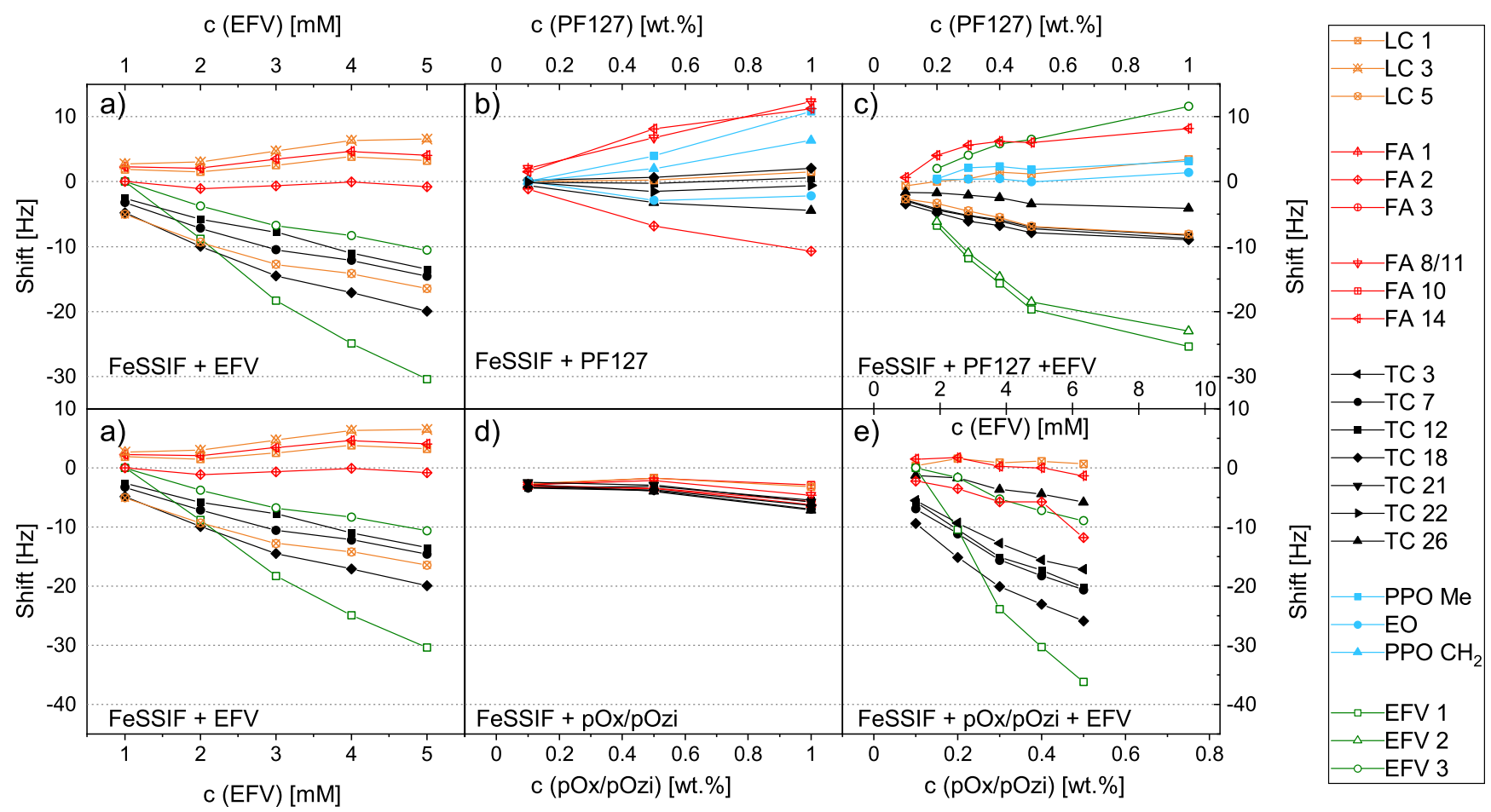

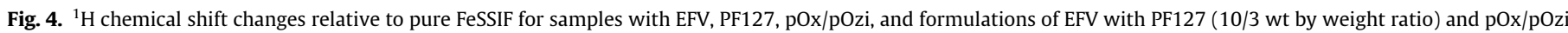

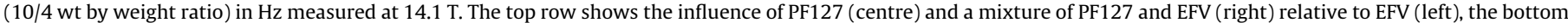

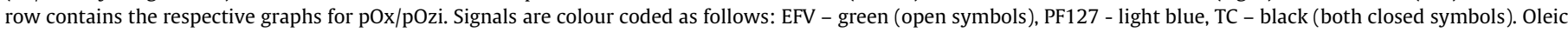

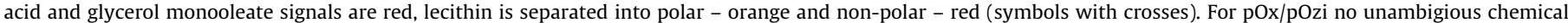

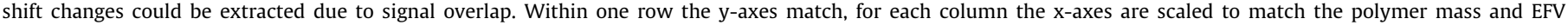
concentration, respectively.

the exchange dynamics between both states averaged or multiple diffusion coefficients can be obtained. This is the case for TC in all samples as it is water soluble but essential for keeping the lipids in solution. This is confirmed by a drop of the TC diffusion coefficient from $3.9 \cdot 10^{-10} \mathrm{~m}^{2} / \mathrm{s}$ ( $\hat{=} 1.5 \mathrm{~nm}$ ) in buffer to $2.3 \cdot 10^{-10} \mathrm{~m}^{2} / \mathrm{s}$ in FeSSIF, which is equivalent to twice the hydrodynamic diameter, but different to the diffusion coefficient of the lipids $\left(6.5 \cdot / \mathrm{s}^{-11} \mathrm{~m}^{2} / \mathrm{s} \hat{=} 8.9 \mathrm{~nm}\right)$. To explain this, it has to be considered that: (i) pure NaTC forms aggregates of 2-5 molecules at concentrations of $10-50 \mathrm{mM}$. [74,75] (ii) NaTC acts as a solubilizer for lecithin in FeSSIF, which is not soluble in water/buffer solutions in the absence of NaTC. This is also confirmed by the slower diffusion coefficient for NaTC in FeSSIF compared to NaTC in buffer. (iii) The exchange of NaTC molecules between NaTC and NaTC-lecithin aggregates is fast on the NMR timescale, which is why an averaged value for NaTC diffusion is observed. Consequently, the exact fraction of NaTC involved in NaTC-lecithin aggregates cannot be determined unambiguously, but the role of NaTC as solubilizer of lecithin is supported by the experiments.

In FeSSIF/EFV samples, the observed diffusion coefficients of lipid components and EFV agreed, which underlines the presence of EFV within the bile colloids. Varying the EFV concentration has no significant impact on the diffusion behaviour, so the particle size was not affected. The summary of diffusion coefficients for all completely dissolved FeSSIF/EFV samples is shown in the SI (Figure S13).

\subsection{Detailed study of PF127 and PF127/EFV in FeSSIF}

The aggregation state of pure PF127 can be straightforwardly examined in ${ }^{1} \mathrm{H}$ NMR spectra using the methyl resonance of PPO, which exhibits fine splittings below the critical micellization tem- perature (CMT)/concentration (CMC) and a broad, shifted resonance above the CMT/CMC as shown in Figure S14. In all mixtures with FeSSIF starting from 0.1\% PF127, micelles were present under the measurement conditions at $37{ }^{\circ} \mathrm{C}$. This is in agreement with a CMT of $31^{\circ} \mathrm{C}$ at the same concentration in pure water published by Alexandridis et al.,[55] even though the micellization properties of Pluronics can be modified by different salt concentrations [76] and interactions with surfactants such as bile acids.[61]

Diffusion experiments of pure PF127 in FeSSIF (Fig. 5, left side) showed that the diffusion coefficients of PF127 and lipids in FeSSIF match indicating the interference of PF127 with the FeSSIF colloids by forming mixed aggregates. Pure PF127 micelles in buffer solution were also significantly larger $\left(D=2.21 \cdot 10^{-11} \mathrm{~m}^{2} / \mathrm{s} \hat{=}\right.$ $26.1 \mathrm{~nm}$ diameter). Furthermore, with increasing amount of polymer the resulting aggregates became larger and diffused more slowly $\left(\mathrm{D}=5.83 \cdot 10^{-11} \mathrm{~m}^{2} / \mathrm{s} \hat{=} 9.9 \mathrm{~nm}, 4.45 \cdot 10^{-11} \mathrm{~m}^{2} / \mathrm{s} \hat{=}\right.$ $13.0 \mathrm{~nm}$ and $2.79 \cdot 10^{-11} \mathrm{~m}^{2} / \mathrm{s}=20.7 \mathrm{~nm}$ at concentration of 0.1 , 0.5 and $1 \mathrm{wt} \%$ PF127, respectively). The estimated mean particle diameter has nearly tripled upon addition of $1 \%$ polymer compared to FeSSIF.

To confirm outcomes from PFG NMR, complementary NOESY spectroscopy with mixing times of $60 \mathrm{~ms}$ was employed. Unlike reported for Pluronic P123, no interactions between the polymer and bile acids can be detected at very low polymer concentrations.[61] However, for PF127 at concentrations of $0.5 \%$ and $1 \%$, contacts to fatty acid chains of lipids were identified (Figure S17). Additionally, variations in cross-peak intensities for TC-TC contacts were observed for FeSSIF/PF127 compared to FeSSIF samples. These observations match the conclusion of mixed aggregates drawn from diffusion data and additionally indicate that these mixed aggregates are highly dynamic such that only weak NOESY signals arise. 


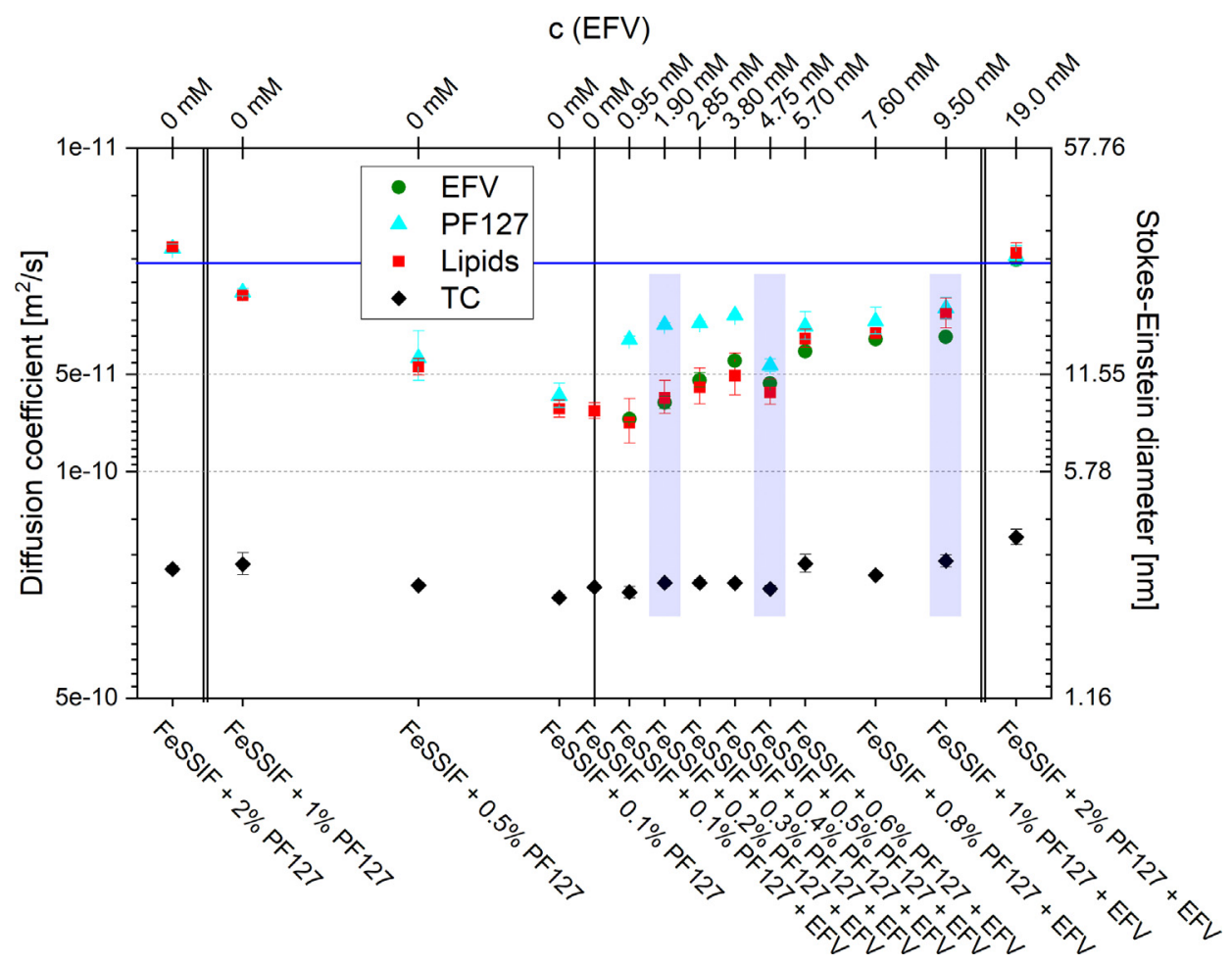

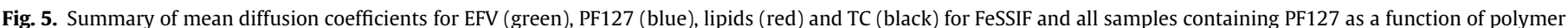

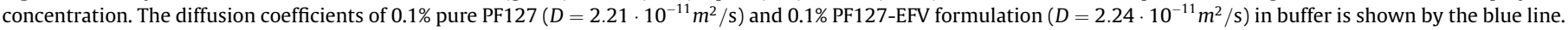

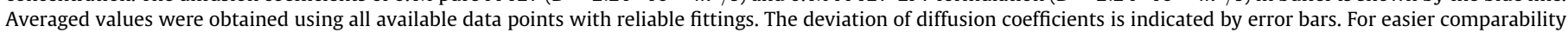

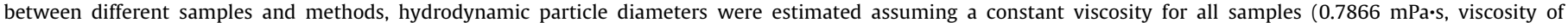
deuterated water at $\left.40{ }^{\circ} \mathrm{C}[72]\right)$. Cryo-TEM images were recorded for mixtures highlighted by blue bars.

As for pure PF127 and in contrast to FeSSIF/EFV (Figure S13), for which diffusion coefficients were not concentration dependent, the addition of a PF127-EFV formulation resulted in decreasing diffusion coefficients and increasing hydrodynamic radii for all components except for TC (Fig. 5, right side). (i) For concentrations below $0.5 \%$ an interesting pattern is observed: EFV and the lipids diffused at the same rate while the polymer exhibited slower diffusion, suggesting a co-existence of larger PF127 micelles and smaller lipidsbased particles. This co-existence collapsed at 0.5\% PF127 with a small decrease in apparent size. With increasing formulation concentration, the aggregates exhibited similar sizes to FeSSIF/PF127 in the absence of EFV. (ii) For concentrations of $0.5 \%$ and higher, the diffusion behaviour of PF127, EFV and lipids became more uniform. Interestingly, this merging of individual particles occurred at the solubility threshold of EFV in FeSSIF. Consequently, the polymer is crucial for keeping the drug solubilized from this concentration onwards. Below 0.5\% polymer, particles consisting mainly of FeSSIF components and EFV seem to be favoured, which shows that the bile salt interfering nature of PF127 is influenced by the interplay of concentration and presence of a small molecule drug. However, it is important to note that the DOSY data only shows the main diffusion coefficient for each component and a smaller amount can still participate in different aggregates or averaged diffusion coefficients may be obtained.

${ }^{1} \mathrm{H}-{ }^{1} \mathrm{H}$ NOESY spectra with short mixing times were acquired for samples with $0.1 \%, 0.4 \%, 0.5 \%$ and $1 \%$ PF127-EFV formulation (Figures S18 and S19). For 1\%, 0.5\% and 0.4\% solutions and $60 \mathrm{~ms}$ mixing time weak contacts between the Pluronic PPO block and FA 8/11/12 could be detected, as well as from both of these moieties to the aromatic proton EFV 3. Additional close contacts of EFV 3 to the three-membered ring (EFV 5/6) as well as COSYtype artefacts with the remaining aromatic resonances at $0.5 \%$ and $1 \%$ were observed. DOSY and NOESY observations can be additionally supported by contacts in 1D 19F-1H HOESY NMR at $0.5 \%$ formulation (Figure S20). At $0.4 \%$ purely absorptive off-diagonal signals were detected between the aromatic EFV protons 1 and 2 . To focus on the environment of EFV, a selective NOESY for the highest concentration (1\%) was acquired by selective excitation of the aromatic protons (Figure S21). Alongside the previously mentioned contacts, weak resonances to FA 10/9 and the hydrophobic side of TC (TC 21 and TC 18) were observed. For the lowest concentration $(0.1 \%)$ and a mixing time of $60 \mathrm{~ms}$ no additional contacts compared to those arising in pure FeSSIF could be observed, apart from an absorptive interaction between EFV protons 1 and 2. Analogous contacts, as observed for concentrations $\geq 0.4 \%$, between EFV or PF127 and other components might be existing but were too weak to be observed. Therefore, the mixing time was set to $1 \mathrm{~s}$ to increase the cross-correlation intensities, while accepting that spin-diffusion will reduce the informative value of specific interactions. This resulted in additional signals between EFV itself and additional contacts of FA 8/11/12 with EFV and PF127, respectively.

From this it can be summarised, that (i) at all concentrations, EFV is located close to the lipids with (ii) a changing mode of interaction between $0.4 \%$ and $0.5 \%$ PF127-EFV formulation. This is observed through the nature of EFV-EFV cross peaks from being absorptive at low concentrations to dispersive at higher concentrations. Again, this coincides with the solubility limit of EFV in FeSSIF and also the merging of different aggregates in DOSY NMR. The interaction of PF127 with lipids at a low concentration of $0.1 \%$ was not expected based on the clearly separated diffusion coefficients of these two molecular entities in the DOSY data indicating separate aggregates. Additionally, the diffusion coefficient of PF127 in the FeSSIF solution with low concentrations of the formulation was significantly higher than for FeSSIF with EFV and the contacts 
between PF127 and FeSSIF are weakly observed at long mixing times. We interpret that (iii) PF127 alone forms larger aggregates (in agreement with DOSY data in buffer solution) and competes with EFV for the FeSSIF components. Although generally forming separate aggregates at lower concentrations, (iv) a small part of PF127 interacts with the lipids/EFV aggregates (identical diffusion
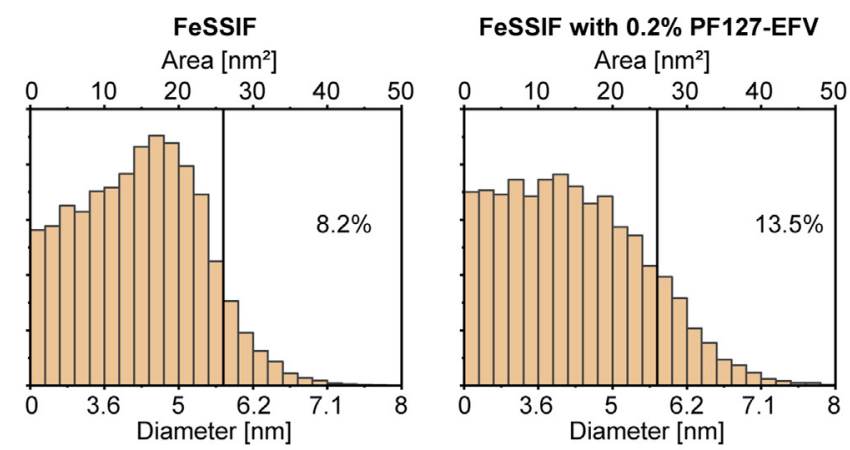

FeSSIF with $0.5 \%$ PF127-EFV Area $\left[\mathrm{nm}^{2}\right]$
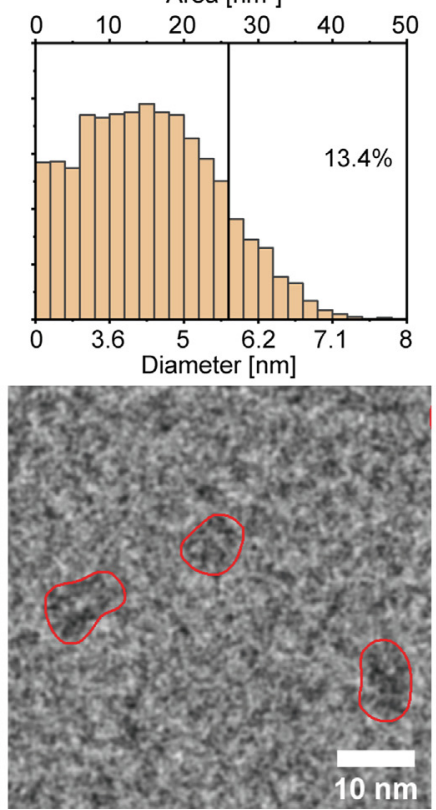

FeSSIF with 1\% PF127-EFV
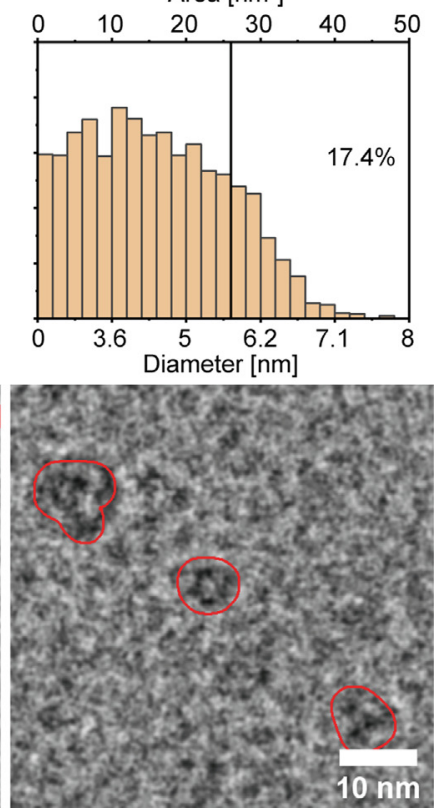

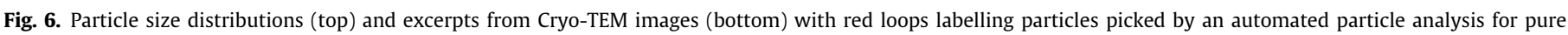

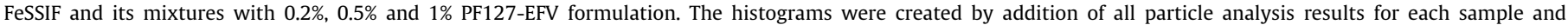
comparable ice thickness. The relative number of particles exceeding diameters of $5.7 \mathrm{~nm}\left(26 \mathrm{~nm}^{2}\right)$ is shown to facilitate comparisons.

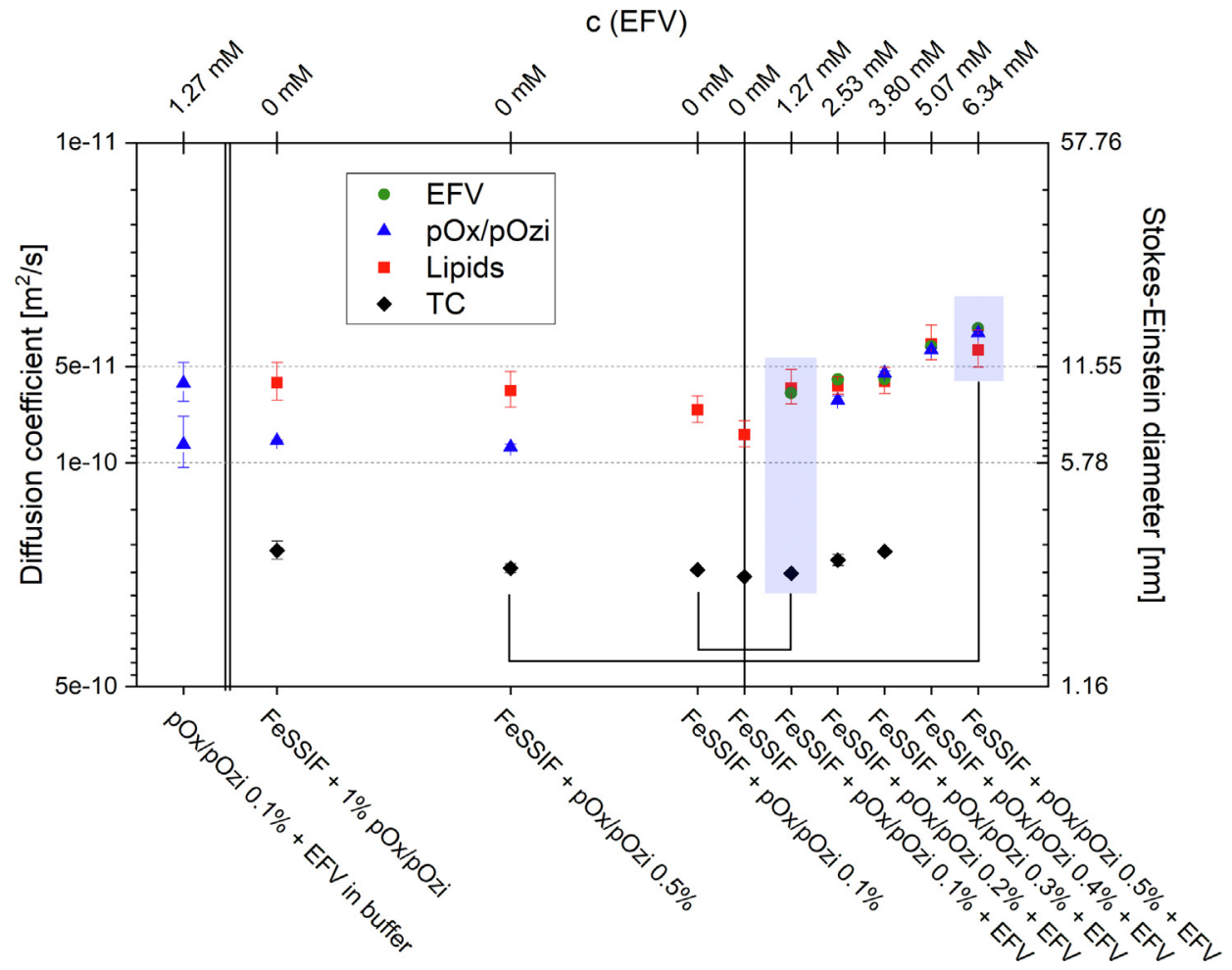

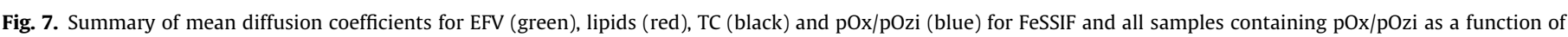

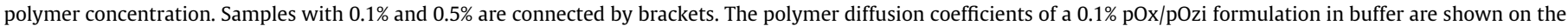

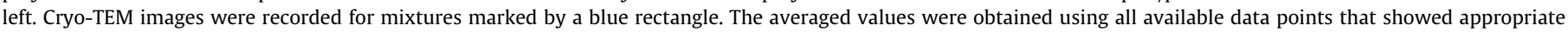

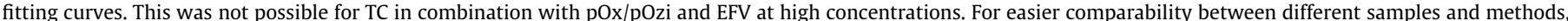

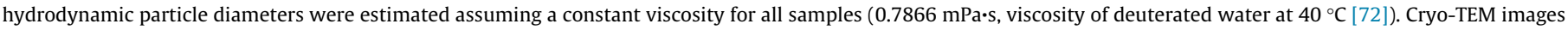
were recorded for mixtures highlighted by blue bars. 
behaviour) on a fast timescale. With increasing formulation concentration, this dynamic interaction increases in line with the slower diffusion of lipids/EFV aggregates prior to the collapse into mixed particles observed at $0.5 \%$ formulation.

To gain orthogonal information on particles sizes, cryo-TEM images were analysed. Generally, it is preferable to have cryoTEM samples with low-salt buffer and minimal additives. The relatively high salt concentrations here and the comparably thick ice layers reduced the contrast between particles and the surroundings, therefore complicating the analysis. All images were inspected visually and also analysed using automatic particle picking to obtain distributions of the particle projection areas that were converted to diameters of circles with equivalent areas. Only particles with a certain roundness were picked excluding clusters of particles. Results were summed for each system and concentration and graphically depicted in histograms. In comparison to NMR spectroscopy, where the intensities are proportional to the number of nuclei in a certain aggregate, the histograms show the overall numbers of particles.

In a first step, pure FeSSIF and FeSSIF with $0.2 \%$ and $0.5 \%$ PF127 were examined (Figures S22). The diameters for pure FeSSIF showed a broad distribution up to $\sim 7.1 \mathrm{~nm}\left(\hat{=} 40 \mathrm{~nm}^{2}\right.$ ) with a maximum at $\sim 4.8 \mathrm{~nm}\left(\hat{=} 18 \mathrm{~nm}^{2}\right)$. This is in good agreement with diameters determined using SAXS (6.5-9.1 nm for FeSSIF-V2, 5.4$6.7 \mathrm{~nm}$ for FeSSIF-V1 at $295 \mathrm{~K}$ ) [77] and molecular dynamics simulations (4-7 nm) [78], but also with the diameter estimated from
PFG experiments in this work $(8.9 \mathrm{~nm})$. In the presence of pure PF127, an increase of particles with diameters exceeding $5.7 \mathrm{~nm}$ ( $\hat{=} 26 \mathrm{~nm}^{2}$ ) could be seen for concentrations of $0.2 \%$ PF127 $(5.95 \%>5.7 \mathrm{~nm})$ and $0.5 \%$ PF127 $(7.19 \%>5.7 \mathrm{~nm})$ compared to FeSSIF $(5.13 \%>5.7 \mathrm{~nm})$. Such an increase in particle size was expected from diffusion data but it is less pronounced than in the cryo-TEM data. A reason for this weaker trend could be that larger particles in cryo-TEM also result in fewer observable particles. Additionally, the estimation of particle sizes based on the Stokes-Einstein equation is prone to errors due to several assumptions made such as the presence of ideal, spherical particles or identical viscosities for all samples. Similar trends for the particle size accompanied by altered shapes of the particle distributions were also observed for mixtures with concentrations of $0.2 \%, 0.5 \%$ and $1 \%$ PF $127-E F V$ formulation (Fig. 6). A coexistence of differently sized particles, as observed by DOSY, is not directly apparent, but the proportion of particles $>5.7 \mathrm{~nm}$ provided more insight. At a concentration of $0.2 \%$ formulation a higher number of larger particles was observed compared to $0.5 \%$. At $1 \%$ the share of large particle diameters is increasing again mirroring the trends observed through DOSY NMR data.

\subsection{Detailed study of pOx/pOzi as well as pOx/pOzi /EFV in FeSSIF}

The triblock copolymer pOx/pOzi shows a CMT of $36{ }^{\circ} \mathrm{C}$ at a concentration of $0.1 \mathrm{wt} \%$.[58] At room temperature, micelles are

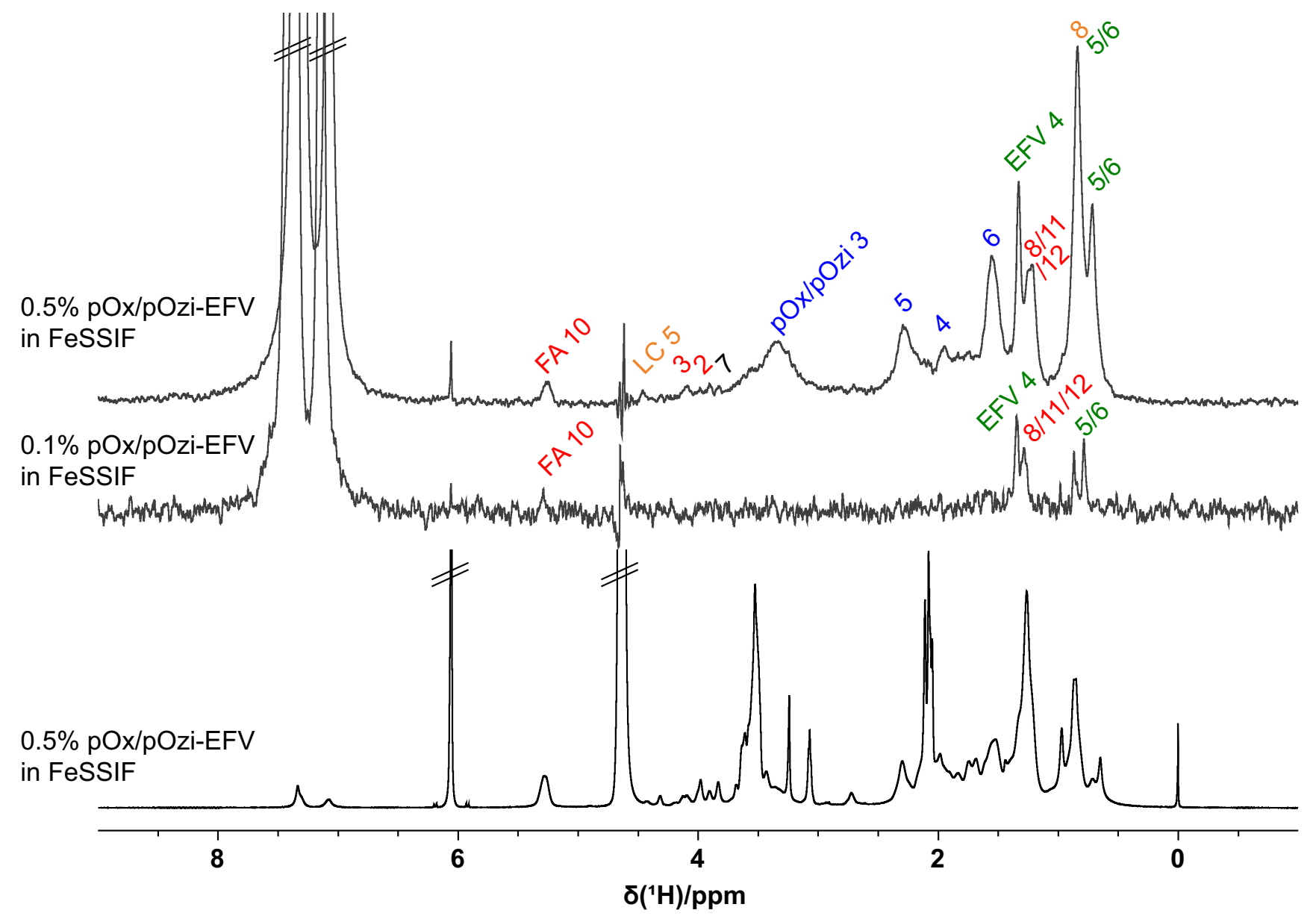

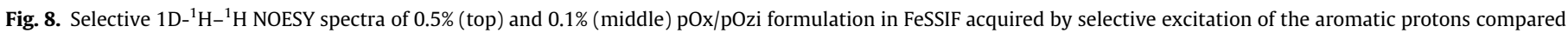

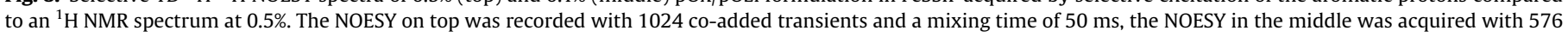

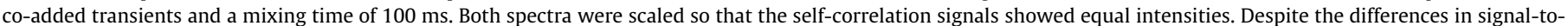

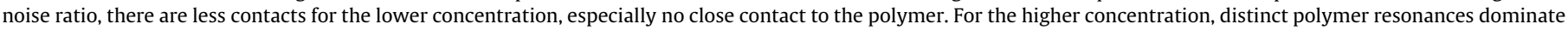
the spectrum at half the mixing time. 
formed in the presence of poorly water-soluble guest molecules and the resulting micelles display solid-like cores. In ${ }^{1} \mathrm{H}$ NMR spectra, this results in extremely wide resonances for hydrophobic guest molecules (FWHM $\sim 300 \mathrm{~Hz} / 0.5$ ppm, Figure S11). A complete assignment of the polymer resonances in water is given in the SI (Figure S8). To confirm the hypothesis that pure pOx/pOzi is not interacting with FeSSIF based on the concentration dependent spectral changes, diffusion coefficients of all components were determined (Fig. 7, left side). For the lowest concentration of $0.1 \%$ polymer no reliable diffusion coefficient could be obtained due to signal overlap of polymer and FeSSIF resonances. At higher polymer concentrations, the signal was dominated by the polymer contribution enabling extraction of diffusion coefficients. Overall, different diffusivities were observed for the lipids and the polymer. This could be due to an averaged diffusion coefficient of pOx/pOzi or due to the presence in different particles. For the highest polymer concentration (1 wt\%), no interactions between FeSSIF and pOx/pOzi could be detected using ${ }^{1} \mathrm{H}-{ }^{1} \mathrm{H}$ NOESY spectroscopy with a mixing time of $60 \mathrm{~ms}$ (Figure S23). This is in agreement with the very small changes in chemical shift observed upon addition polymer to FeSSIF, from which it can be deduced, that pOx/pOzi does not interfere or interact in any meaningful manner with the bile colloids.

Interestingly, in the summarized diffusion coefficients for FeSSIF with pOx/pOzi EFV formulations (Fig. 7, right side) a significant decrease in diffusion coefficients and increase in hydrodynamic radii was observed with increasing formulation concentration. For identical polymer concentrations, overall larger particle sizes were observed for the formulations compared to pure polymer in FeSSIF (black brackets in Fig. 7). In contrast to the mixtures with pure polymer, the polymer exhibited the same diffusivity as EFV and the lipids indicating the presence of mixed particles. For low formulation concentrations, TC diffusion was almost constant. However, for concentrations above $0.3 \%$, the TC attenuation curves showed an additional curvature requiring fitting with a biexponential instead of a mono-exponential fit. Diffusion parameters could be extracted as $4.3 \cdot 10^{-11} \pm 6.8 \cdot 10^{-12} \mathrm{~m}^{2} / \mathrm{s}(\hat{=} 13.4 \mathrm{~nm})$ and $2.2 \cdot 10^{-10} \pm 1.4 \cdot 10^{-11} \mathrm{~m}^{2} / \mathrm{s}(\hat{=} 2.6 \mathrm{~nm})$ for a $0.4 \%$ concentration of the formulation. As they are not separated by an order of magnitude, they are less reliable and thus not included in the figure. However, this change in curvature and the diffusion rates close to the lipids indicate that mixed particles with slower exchange of TC exist at higher formulation concentrations.

Complementary spatial proximities though NOE data were also examined for mixtures containing pOx/pOzi formulations. At a mixing time of just $20 \mathrm{~ms}$, multiple cross-correlations are present for the highest concentration of $0.5 \%$ (Figure S24), which is surprising as nearly no close contacts were measured under similar conditions for PF127 formulations. This could be due to comparably rigid aggregates. Specifically, proximity of EFV to both lipids and TC as well as pOx/pOzi is observed. Moreover, the intensity and number of TC-TC contacts increased, which supports the hypothesis of slower TC exchange in the particles. Upon closer examination by selective NOESY (Fig. 8, selective excitation of aromatic EFV protons, mixing time of $50 \mathrm{~ms}$ ) additional proximities from EFV to the hydrophobic pOzi block can be confirmed.

Spectra with similar appearance were obtained for concentrations $0.4 \%$ and $0.3 \%$, but interestingly, a different behaviour was observed for the lowest concentration (0.1\%, Figure S25). Here, no spatial proximities to EFV could be identified. This is partially
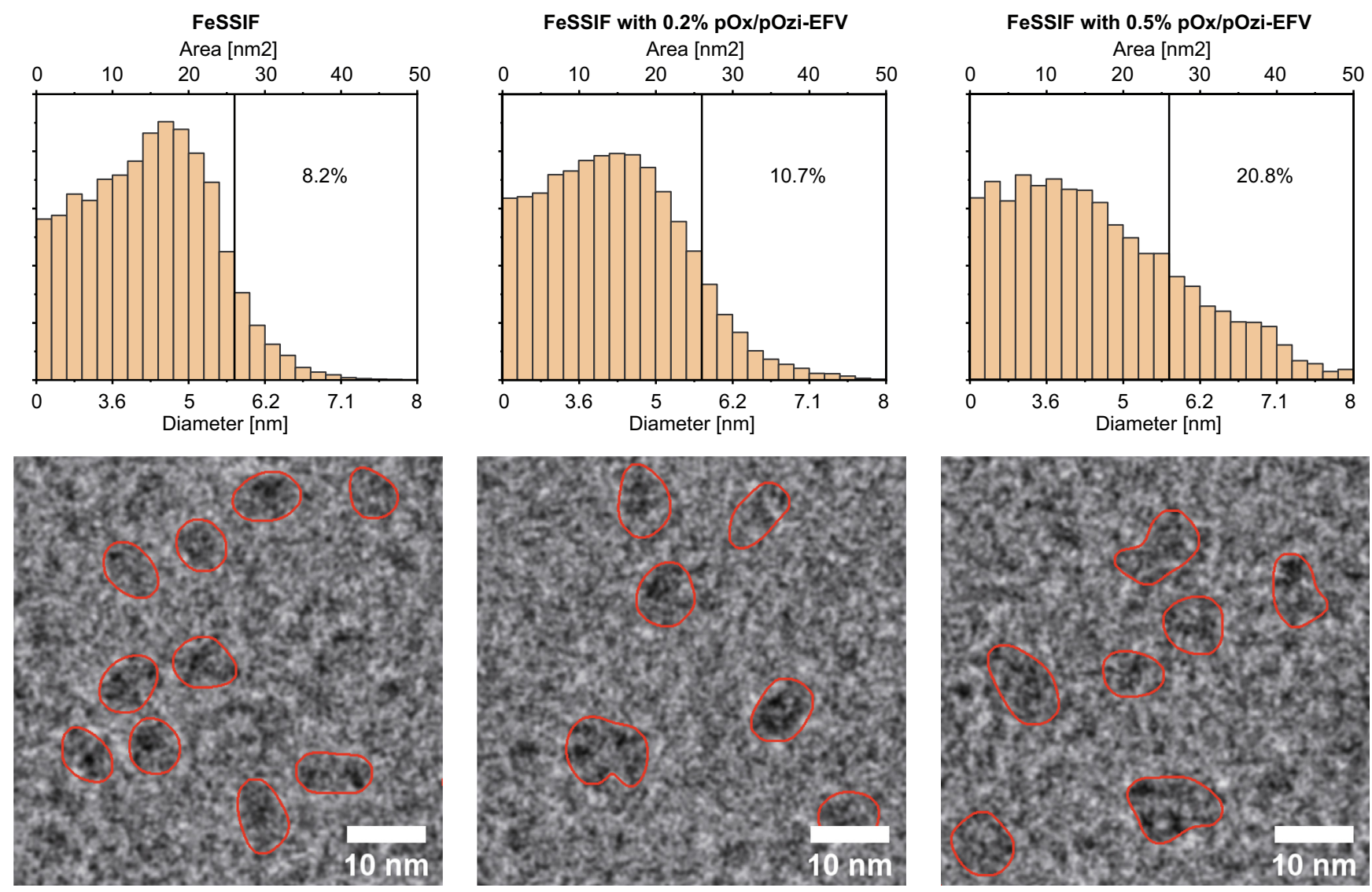

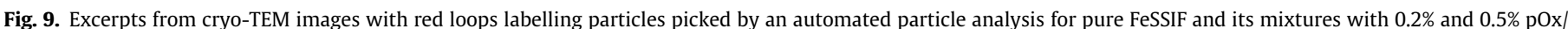

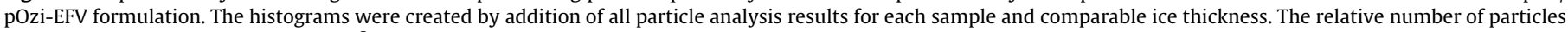
exceeding diameters of $5.7 \mathrm{~nm}\left(26 \mathrm{~nm}^{2}\right)$ is shown to facilitate comparisons. 
an effect due to the lower overall EFV concentration, but even in selective NOESY experiments with mixing times of $100 \mathrm{~ms}$ and $200 \mathrm{~ms}$ only contacts between EFV and FA8/11/12 could be detected. This difference in dipolar transfer rates in NOE experiments is among other factors dependent on the mean distance between interacting dipoles during the mixing time,[79] which for aggregates is strongly dependent on the mobility and exchange dynamics of the components. In the case of pOx/pOzi formulations these dynamics seem to strongly depend on the amount of polymer. At low concentrations EFV resonances could be detected in ${ }^{1} \mathrm{H}$ NMR data, which is not the case in pure buffer solution and even multiplets due to J-coupling could be observed. With increasing formulation concentration these resonances coalesced, and peak widths broaden for all components. In general, EFV resonances were only visible when they were partially solubilized by TC and lipids. With increasing amounts of formulation, the relative proportion of these solubilizers decreases, more similar aggregates to the formulation without FeSSIF and more rigid aggregates are formed. This agrees with an increased number of TC-TC contacts and the different attenuation curves in the diffusion data.

Based on DOSY data in combination with NOESY contacts two scenarios are possible for the concentration dependent behaviour of pOx/pOzi EFV formulations in FeSSIF: (1) All components are contributing to mixed particles in agreement with an equal diffusion behaviour of $\mathrm{pOx} / \mathrm{pOzi}$, lipids and EFV and supported by a size increase of the observed aggregates compared to EFV/FeSSIF and pOx/pOzi/FeSSIF. (2) There could be separate pOx/pOzi/EFV particles, which interact weakly with TC. This could result in larger lipid particles due to the lack of available solubilizing TC molecules. Although this could explain the unusual attenuation curves observed for TC at higher formulation concentrations, this would imply that the diffusion coefficients of three different species
(pOx/pOzi, lipids, EFV) accidentally coincide, therefore making scenario (1) more probable.

Cryo-TEM gives additional insights into particle sizes and shapes. Samples with $0.2 \%$ and $0.5 \%$ pOx/pOzi-EFV formulation (Fig. 7, blue bars) were analysed with respect to the distribution of particle projection areas. pOx/pOzi formulations showed slower diffusion at a higher polymer content in the DOSY data. This behaviour is also mirrored in the corresponding cryo-TEM images. The histograms (Fig. 9) showed a shift to larger particle projection areas from pure FeSSIF $(8.2 \%>5.7 \mathrm{~nm}$ over $0.2 \%(10.7 \%>$ $5.7 \mathrm{~nm})$ to $0.5 \%$ formulation content $(20.8 \%>5.7 \mathrm{~nm})$. Furthermore, the overall shape of the distribution also changed, indicating a broader distribution of different particle sizes as compared to more uniform particle sizes found for FeSSIF. This agrees with fittings of the polymer attenuation curves to a log-normal distribution. With increasing formulation concentration, the parameter representing the width of the log normal distribution increased as well.

\section{Conclusion}

The behaviour of two triblock copolymers, PF127 and a pOx/ pOzi, as well as their formulations with the HIV-drug efavirenz in biorelevant media simulating the fed state intestinal fluids (FeSSIF) was investigated in detail using NMR spectroscopy in solution complemented by cryo-TEM. Interestingly, for both polymers, the behaviour differed between pure polymer and the formulation in FeSSIF and is schematically summarized in Fig. 10. While the polymers alone could be straightforwardly classified as bile interacting polymers (PF127) and bile non-interacting (pOx/pOzi) based on ${ }^{1} \mathrm{H}$ NMR spectra and observations from DOSY experiments, the situa-
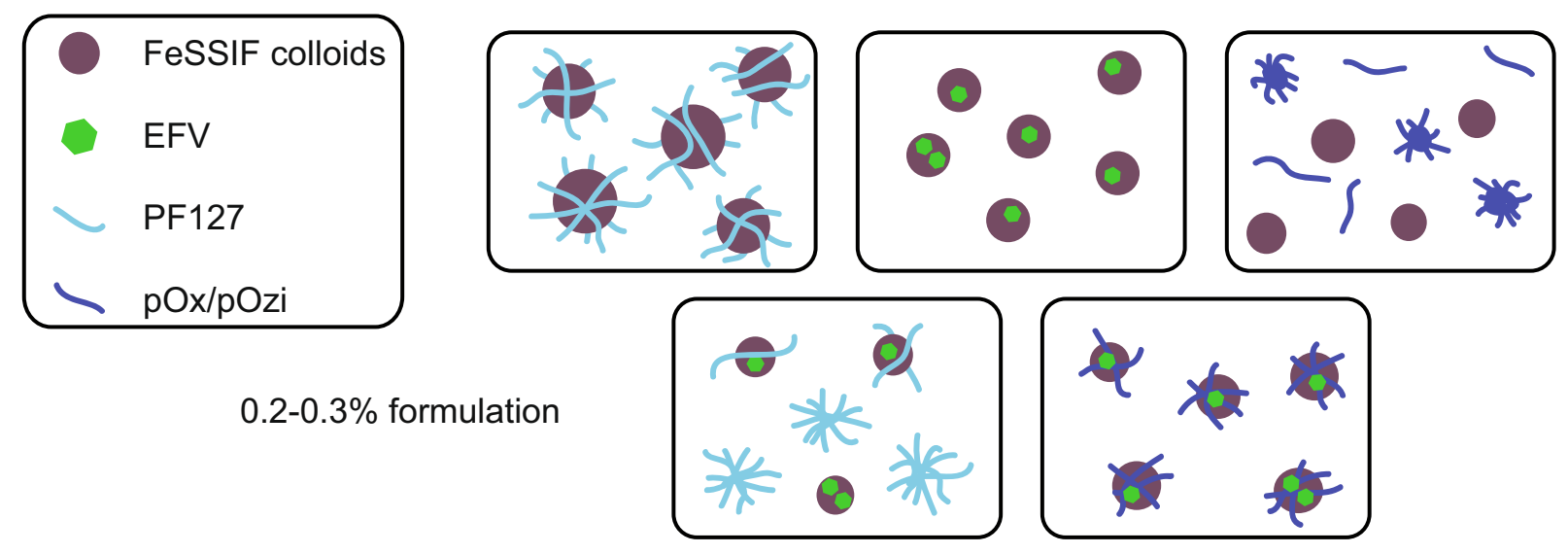

$0.2-0.3 \%$ formulation

$0.5 \%$ formulation
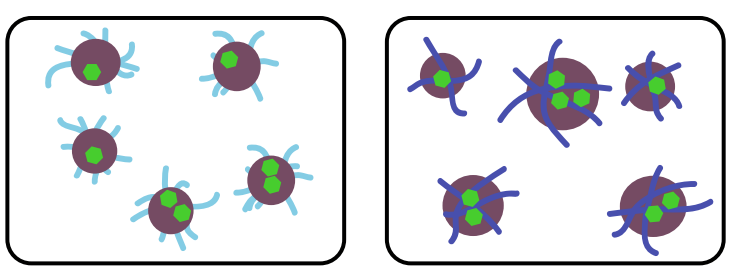

$1 \%$ formulation

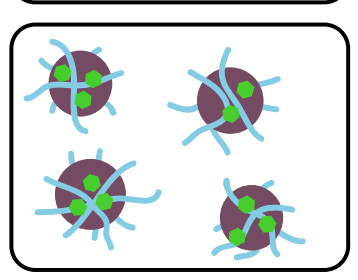

Fig. 10. Schematic summary of changes in aggregation behaviour dependent on solute and solute concentration. 
tion is more complex when the formulations were part of these multicomponent mixtures. In this case, the bile interacting nature was observed to depend on both the polymer/formulations concentration and the presence of the small molecule drug. Using diffusion NMR, through space proximities and cryo-TEM measurements helped to assemble the following picture: For PF127/EFV formulations in FeSSIF coexisting separated particles were observed for formulation concentrations $<0.4 \mathrm{wt} \%$. Above this threshold, a collapse of these particles is observed, which subsequently diffuse with a joint diffusion coefficient, which decreases upon further increasing the concentration. Even though individually non-interfering with bile colloids, pOx/pOzi /EFV formulations do show aggregation phenomena with bile colloids as indicated by coinciding concentration dependent diffusion coefficients of the polymer, EFV and lipids. This shows that careful and case-by-case analysis that also considers the drug is required with respect to classification of polymers regarding their bile interfering nature. This details our previous report, in that screening of individual, plain polymers in FeSSIF may not be sufficient to account for the multifaceted behaviour of polymer-drug formulations in simulating intestinal fluids as indicated here for two polymers with the model drug efavirenz.[41] This could also have implications for patients with co-morbidities taking multiple pharmaceutical products. They could form a complex multicomponent mixture in the intestinal tract not just with respect to potential drug-drug interferences but also with respect to polymeric excipients. The present study showcases that through this set of analytical tools valuable insights into the behaviour of polymer-drug formulations in biorelevant media can be obtained. In further studies, more formulations and also drug loading dependent features, but also more sophisticated biorelevant environments such as material obtained from intestinal biopsies need to be analysed to improve our understanding of such colloidal aggregates and the implications for the design of future pharmaceutical products.

\section{CRediT authorship contribution statement}

Sebastian Endres: Conceptualization, Methodology, Software, Formal analysis, Investigation, Data curation, Writing - original draft, Writing - review \& editing, Visualization. Emil Karaev: Investigation. Simon Hanio: Writing - review \& editing, Validation. Jonas Schlauersbach: Writing - review \& editing, Validation. Christian Kraft: Investigation. Tim Rasmussen: Data curation, Validation. Robert Luxenhofer: Resources, Writing - review \& editing. Bettina Böttcher: Resources, Validation, Writing - review \& editing. Lorenz Meinel: Resources, Writing - review \& editing, Supervision. Ann-Christin Pöppler: Writing - review \& editing, Methodology, Resources, Supervision, Conceptualization, Project administration, Funding acquisition.

\section{Declaration of Competing Interest}

The authors declare that they have no known competing financial interests or personal relationships that could have appeared to influence the work reported in this paper.

\section{Acknowledgement}

We thank Matthias Grüne for helpful discussion and his support during data collection. M. M. Lübtow kindly provided the $\mathrm{pOx} / \mathrm{pOzi}$ EFV formulation. We gratefully acknowledge funding by the German Research Foundation (DFG) project number 440955393. Financial support from the Verband der Chemischen Industrie (VCI) in the form of a material cost allowance as well as from the University of Würzburg in the form of a CNC PostDoc Plus allow- ance (financed from Emil-Hilb-funds) is also acknowledged (A.-C. P.). Electron microscopic data were acquired at the cryo-EM facility in Würzburg (DFG Equipment Grant INST 93/903-1 FUGG). Raw experimental data is available through the repository Zenodo via https://doi.org/10.5281/zenodo.5136465.

\section{Appendix A. Supplementary material}

Supplementary data to this article can be found online at https://doi.org/10.1016/j.jcis.2021.08.040.

\section{References}

[1] L.Z. Benet, The Role of BCS (Biopharmaceutics Classification System) and BDDCS (Biopharmaceutics Drug Disposition Classification System) in Drug Development, J. Pharm. Sci. 102 (1) (2013) 34-42.

[2] I. Nikolakakis, I. Partheniadis, Self-emulsifying granules and pellets: composition and formation mechanisms for instant or controlled release, Pharmaceutics 9 (4) (2017) 50.

[3] T.M. Allen, P.R. Cullis, Drug delivery systems: entering the mainstream 1818 , Science 303 (5665) (2004).

[4] Editorial: Drug Delivery: Too Much Complexity, Not Enough Reproducibility? Angew Chem. Int. Ed. 56 (48) (2017) 15170-15171.

[5] V.J. Venditto, F.C. Szoka Jr., Cancer nanomedicines: so many papers and so few drugs! 80, Adv Drug Delivery Rev. 65 (1) (2013).

[6] R. Luxenhofer, Polymers and nanomedicine: considerations on variability and reproducibility when combining complex systems, Nanomedicine 10 (20) (2015) 3109-3119.

[7] J. Kost, R. Langer, Responsive polymeric delivery systems, Adv. Drug Delivery Rev. 64 (2012) 327-341.

[8] X. Sang, Q. Yang, G. Shi, L. Zhang, D. Wang, C. Ni, Preparation of pH/redox dual responsive polymeric micelles with enhanced stability and drug controlled release, Mater. Sci. Eng. C 91 (2018) 727-733.

[9] X. Guo, Y. Cheng, X. Zhao, Y. Luo, J. Chen, W.-E. Yuan, Advances in redoxresponsive drug delivery systems of tumor microenvironment, J. Nanobiotechnol. 16 (1) (2018) 74.

[10] T.L. Rapp, C.A. DeForest, Targeting drug delivery with light: A highly focused approach, Adv. Drug Delivery Rev. 171 (2021) 94-107.

[11] A. Bratek-Skicki, Towards a new class of stimuli-responsive polymer-based materials - Recent advances and challenges, Appl. Surf. Sci. Adv. 4 (2021) 100068.

[12] M. Yokoyama, S. Inoue, K. Kataoka, N. Yui, Y. Sakurai, Preparation of adriamycin-conjugated poly(ethylene glycol)-poly(aspartic acid) block copolymer. A new type of polymeric anticancer agent, Macromol. Rapid Commun. 8 (9) (1987) 431-435.

[13] I. Ekladious, Y.L. Colson, M.W. Grinstaff, Polymer-drug conjugate therapeutics: advances, insights and prospects, Nat. Rev, Drug Discovery, 2018.

[14] F. Farjadian, A. Ghasemi, O. Gohari, A. Roointan, M. Karimi, M.R. Hamblin, Nanopharmaceuticals and nanomedicines currently on the market: challenges and opportunities, Nanomedicine 14 (1) (2018) 93-126.

[15] C.H. Wong, K.W. Siah, A.W. Lo, Estimation of clinical trial success rates and related parameters, Biostatistics 20 (2) (2018) 273-286.

[16] P. Bannigan, J. Flynn, S.P. Hudson, The impact of endogenous gastrointestinal molecules on the dissolution and precipitation of orally delivered hydrophobic APIs, Expert Opin. Drug Delivery 17 (5) (2020) 677-688.

[17] B.S. Prachi, B.P. Varsha, Understanding peroral absorption: regulatory aspects and contemporary approaches to tackling solubility and permeability hurdles 260, Acta Pharm. Sin. B 7 (3) (2017).

[18] N. Pavurala, L.E.K. Achenie, A mechanistic approach for modeling oral drug delivery, Comput. Chem. Eng. 57 (2013) 196-206.

[19] T. Takagi, T. Masada, K. Minami, M. Kataoka, K.-I. Izutsu, K. Matsui, S. Yamashita, In Vitro Sensitivity Analysis of the Gastrointestinal Dissolution Profile of Weakly Basic Drugs in the Stomach-to-Intestine Fluid Changing System: Explanation for Variable Plasma Exposure after Oral Administration, Mol. Pharmaceutics 18 (4) (2021) 1711-1719.

[20] J. Stappaerts, B. Wuyts, J. Tack, P. Annaert, P. Augustijns, Human and simulated intestinal fluids as solvent systems to explore food effects on intestinal solubility and permeability, Eur. J. Pharm. Sci. 63 (2014) 178-186.

[21] P.A. Elvang, A.H. Hinna, J. Brouwers, B. Hens, P. Augustijns, M. Brandl, Bile Salt Micelles and Phospholipid Vesicles Present in Simulated and Human Intestinal Fluids: Structural Analysis by Flow Field-Flow Fractionation/Multiangle Laser Light Scattering, J. Pharm. Sci. 105 (9) (2016) 2832-2839.

[22] K. Pyper, J. Brouwers, P. Augustijns, I. Khadra, C. Dunn, C.G. Wilson, G.W. Halbert, Multidimensional analysis of human intestinal fluid composition, Eur. J. Pharm. Biopharm. 153 (2020) 226-240.

[23] B.J. Aungst, Novel Formulation Strategies for Improving Oral Bioavailability of Drugs with Poor Membrane Permeation or Presystemic Metabolism, J. Pharm. Sci. 82 (10) (1993) 979-987.

[24] G.L. Amidon, H. Lennernäs, V.P. Shah, J.R. Crison, A Theoretical Basis for a Biopharmaceutic Drug Classification: The Correlation of in Vitro Drug Product Dissolution and in Vivo Bioavailability, Pharm. Res. 12 (3) (1995) 413-420. 
[25] J.B. Dressman, G.L. Amidon, C. Reppas, V.P. Shah, Dissolution Testing as a Prognostic Tool for Oral Drug Absorption: Immediate Release Dosage Forms, Pharm. Res. 15 (1) (1998) 11-22.

[26] J.H. Fagerberg, O. Tsinman, N. Sun, K. Tsinman, A. Avdeef, C.A.S. Bergström, Dissolution Rate and Apparent Solubility of Poorly Soluble Drugs in Biorelevant Dissolution Media, Mol. Pharmaceutics 7 (5) (2010) 1419-1430.

[27] S. Kollipara, R.K. Gandhi, Pharmacokinetic aspects and in vitro-in vivo correlation potential for lipid-based formulations, Acta Pharm. Sin. B 4 (5) (2014) 333-349.

[28] M. Vogtherr, A. Marx, A.-C. Mieden, C. Saal, Investigation of solubilising effects of bile salts on an active pharmaceutical ingredient with unusual $\mathrm{pH}$ dependent solubility by NMR spectroscopy, Eur. J. Pharm. Biopharm. 92 (2015) 32-41.

[29] Z. Zhou, C. Dunn, I. Khadra, C.G. Wilson, G.W. Halbert, Influence of Physiological Gastrointestinal Surfactant Ratio on the Equilibrium Solubility of BCS Class II Drugs Investigated Using a Four Component Mixture Design, Mol. Pharmaceutics 14 (12) (2017) 4132-4144.

[30] K. Forner, C. Roos, D. Dahlgren, F. Kesisoglou, M.A. Konerding, J. Mazur, H. Lennernäs, P. Langguth, Optimization of the Ussing chamber setup with excised rat intestinal segments for dissolution/permeation experiments of poorly soluble drugs, Drug Dev. Ind. Pharm. 43 (2) (2017) 338-346.

[31] N. Bou-Chacra, K.J.C. Melo, I.A.C. Morales, E.S. Stippler, F. Kesisoglou, M. Yazdanian, R. Löbenberg, Evolution of Choice of Solubility and Dissolution Media After Two Decades of Biopharmaceutical Classification System, The AAPS Journal 19 (4) (2017) 989-1001.

[32] Y.E. Arnold, J. Thorens, S. Bernard, Y.N. Kalia, Drug transport across porcine intestine using an ussing chamber system: regional differences and the effect of P-Glycoprotein and CYP3A4 activity on drug absorption, Pharmaceutics 11 (3) (2019) 139.

[33] K. Patel, R. Doddapaneni, M. Patki, V. Sekar, A. Bagde, M. Singh, ErlotinibValproic Acid Liquisolid Formulation: Evaluating Oral Bioavailability and Cytotoxicity in Erlotinib-Resistant Non-small Cell Lung Cancer Cells, AAPS PharmSciTech 20 (3) (2019) 135.

[34] C. Roos, D. Dahlgren, E. Sjögren, M. Sjöblom, M. Hedeland, H. Lennernäs, Effects of absorption-modifying excipients on jejunal drug absorption in simulated fasted and fed luminal conditions, Eur. J. Pharm. Biopharm. 142 (2019) 387395.

[35] M. Chegireddy, G.K. Hanegave, D. Lakshman, A. Urazov, K.N. Sree, S.A. Lewis, S. J. Dengale, The Significance of Utilizing In Vitro Transfer Model and Media Selection to Study the Dissolution Performance of Weak Ionizable Bases: Investigation Using Saquinavir as a Model Drug, AAPS PharmSciTech 21 (2) (2020) 47.

[36] D. Riethorst, P. Baatsen, C. Remijn, A. Mitra, J. Tack, J. Brouwers, P. Augustijns, An In-Depth View into Human Intestinal Fluid Colloids: Intersubject Variability in Relation to Composition, Mol. Pharmaceutics 13 (10) (2016) 3484-3493.

[37] N.M. Zaki, P. Artursson, C.A.S. Bergström, A Modified Physiological BCS for Prediction of Intestinal Absorption in Drug Discovery, Mol. Pharmaceutics 7 (5) (2010) 1478-1487.

[38] A. Roy, N. Kundu, D. Banik, J. Kuchlyan, N. Sarkar, How does bile salt penetration affect the self-assembled architecture of pluronic P123 micelles? light scattering and spectroscopic investigations, Phys. Chem. Chem. Phys. 17 (30) (2015) 19977-19990.

[39] S. Bayati, L. Galantini, K.D. Knudsen, K. Schillén, Complexes of PEO-PPO-PEO triblock copolymer P123 and bile salt sodium glycodeoxycholate in aqueous solution: A small angle X-ray and neutron scattering investigation, Colloids Surf, A 504 (2016) 426-436.

[40] V. Patel, B. Bharatiya, D. Ray, V.K. Aswal, P. Bahadur, Investigations on microstructural changes in $\mathrm{pH}$ responsive mixed micelles of Triton X-100 and bile salt, J. Colloid Interface Sci. 441 (2015) 106-112.

[41] J. Schlauersbach, S. Hanio, B. Lenz, S.P.B. Vemulapalli, C. Griesinger, A.-C. Pöppler, C. Harlacher, B. Galli, L. Meinel, Leveraging bile solubilization of poorly water-soluble drugs by rational polymer selection, J. Control. Release 330 (2021) 36-48.

[42] S.T. Buckley, K.J. Frank, G. Fricker, M. Brandl, Biopharmaceutical classification of poorly soluble drugs with respect to "enabling formulations", Eur. J. Pharm. Sci. 50 (1) (2013) 8-16.

[43] S. Hanio, J. Schlauersbach, B. Lenz, F. Spiegel, R.A. Böckmann, R. Schweins, I. Nischang, U.S. Schubert, S. Endres, A.-C. Pöppler, F.P. Brandl, T.M. Smit, K. Kolter, L. Meinel, Drug-Induced Dynamics of Bile Colloids, Langmuir 37 (8) (2021) 2543-2551.

[44] B. Cote, D. Rao, A.W.G. Alani, Nanomedicine for Drug Delivery throughout the Alimentary Canal, Mol. Pharmaceutics, 2021.

[45] M. Petrusevska, M. Homar, B. Petek, A. Resman, D. Kocjan, U. Urleb, L. Peternel, Hydroxypropyl Methylcellulose Mediated Precipitation Inhibition of Sirolimus: From a Screening Campaign to a Proof-of-Concept Human Study, Mol. Pharmaceutics 10 (6) (2013) 2299-2310.

[46] J. Wiest, M. Saedtler, B. Böttcher, M. Grüne, M. Reggane, B. Galli, U. Holzgrabe, L. Meinel, Geometrical and Structural Dynamics of Imatinib within Biorelevant Colloids, Mol. Pharmaceutics 15 (10) (2018) 4470-4480.

[47] Z. Vinarov, V. Katev, N. Burdzhiev, S. Tcholakova, N. Denkov, Effect of Surfactant-Bile Interactions on the Solubility of Hydrophobic Drugs in Biorelevant Dissolution Media, Mol. Pharmaceutics 15 (12) (2018) 5741-5753.

[48] C. Pigliacelli, P. Belton, P. Wilde, S. Qi, Probing the molecular interactions between pharmaceutical polymeric carriers and bile salts in simulated gastrointestinal fluids using NMR spectroscopy, J. Colloid Interface Sci. 551 (2019) 147-154.

[49] D.A. Chiappetta, C. Hocht, C. Taira, A. Sosnik, Oral pharmacokinetics of the antiHIV efavirenz encapsulated within polymeric micelles, Biomaterials 32 (9) (2011) 2379-2387.

[50] R. Cristofoletti, A. Nair, B. Abrahamsson, D.W. Groot, S. Kopp, P. Langguth, J.E. Polli, V.P. Shah, J.B. Dressman, Biowaiver Monographs for Immediate Release Solid Oral Dosage Forms: Efavirenz, J. Pharm. Sci. 102 (2) (2013) 318-329.

[51] S.M.E. Vrouenraets, F.W.N.M. Wit, J.v. Tongeren, J.M.A. Lange, Efavirenz: a review, Expert Opin. Pharmacother. 8 (6) (2007) 851-871.

[52] Zênia Maria Maciel Lavra, Davi Pereira de Santana, Maria Inês Ré, Solubility and dissolution performances of spray-dried solid dispersion of Efavirenz in Soluplus, Drug Dev. Ind. Pharm. 43 (1) (2017) 42-54.

[53] Diego A Chiappetta, Christian Hocht, Carlos Taira, Alejandro Sosnik, Efavirenzloaded polymeric micelles for pediatric anti-HIV pharmacotherapy with significantly higher oral bioavailability, Nanomedicine 5 (1) (2010) 11-23.

[54] H.M. Mansour, M. Sohn, A. Al-Ghananeem, P.P. Deluca, Materials for pharmaceutical dosage forms: molecular pharmaceutics and controlled release drug delivery aspects, Int. J. Mol. Sci. 11 (9) (2010) 3298-3322.

[55] P. Alexandridis, J.F. Holzwarth, T.A. Hatton, Micellization of poly (ethylene oxide)-poly (propylene oxide)-poly (ethylene oxide) triblock copolymers in aqueous solutions: thermodynamics of copolymer association, Macromolecules 27 (9) (1994) 2414-2425.

[56] T. Lorson, M.M. Lübtow, E. Wegener, M.S. Haider, S. Borova, D. Nahm, R. Jordan, M. Sokolski-Papkov, A.V. Kabanov, R. Luxenhofer, Poly(2-oxazoline)s based biomaterials: A comprehensive and critical update, Biomaterials 178 (2018) 204-280.

[57] Ondrej Sedlacek, Richard Hoogenboom, Drug Delivery Systems Based on Poly (2-Oxazoline)s and Poly(2-Oxazine)s, Adv. Ther. 3 (1) (2020) 1900168, https:// doi.org/10.1002/adtp.v3.110.1002/adtp.201900168.

[58] M.M. Lübtow, L.C. Nelke, J. Seifert, J. Kühnemundt, G. Sahay, G. Dandekar, S.L. Nietzer, R. Luxenhofer, Drug induced micellization into ultra-high capacity and stable curcumin nanoformulations: Physico-chemical characterization and evaluation in 2D and 3D in vitro models, J. Control Release 303 (2019) 162180.

[59] L. Michael M, H. Malik Salman, K. Marius, K. Stefanie, L. Robert, Like Dissolves Like? A Comprehensive Evaluation of Partial Solubility Parameters to Predict Polymer-Drug Compatibility in Ultra-High Drug Loaded Polymer Micelles, 2019.

[60] Michael M. Lübtow, Malik Salman Haider, Marius Kirsch, Stefanie Klisch, Robert Luxenhofer, Like Dissolves Like?A Comprehensive Evaluation of Partial Solubility Parameters to Predict Polymer-Drug Compatibility in Ultrahigh Drug-Loaded Polymer Micelles, Biomacromolecules 20 (8) (2019) 3041-3056.

[61] Vijay Patel, Debes Ray, Anita Bahadur, Junhe Ma, V.K. Aswal, Pratap Bahadur, Pluronic ${ }^{\circledR}$-bile salt mixed micelles, Colloid. Surface. B 166 (2018) 119-126.

[62] A.J. Clulow, B. Barber, M. Salim, T. Ryan, B.J. Boyd, Synergistic and antagonistic effects of non-ionic surfactants with bile salt + phospholipid mixed micelles on the solubility of poorly water-soluble drugs, Int. J. Pharm. 588 (2020) 119762.

[63] F.G.K. Baucke, Further Insight into the Dissociation Mechanism of Glass Electrodes. The Response in Heavy Water, J. Phys. Chem. B 102 (24) (1998) 4835-4841.

[64] Julie C. Adkins, Stuart Noble, Efavirenz, Drugs 56 (6) (1998) 1055-1064.

[65] Deanna M. Mudie, Gordon L. Amidon, Gregory E. Amidon, Physiological parameters for oral delivery and in vitro testing, Mol. Pharmaceutics 7 (5) (2010) 1388-1405.

[66] Luca Marciani, Eleanor F. Cox, Caroline L. Hoad, Susan Pritchard, John J. Totman, Steve Foley, Amisha Mistry, Steven Evans, Penny A. Gowland, Robin C. Spiller, Postprandial Changes in Small Bowel Water Content in Healthy Subjects and Patients With Irritable Bowel Syndrome, Gastroenterology 138 (2) (2010) 469-477.e1.

[67] E Placidi, C L Hoad, L Marciani, P A Gowland, R C Spiller, PTH-045 Effects of an osmotic laxative on the distribution of water between the small and large intestine in humans, Gut 59 (Suppl 1) (2010) A141.1-A141.

[68] D.H. Wu, A.D. Chen, C.S. Johnson, An Improved Diffusion-Ordered Spectroscopy Experiment Incorporating Bipolar-Gradient Pulses, J. Magn. Reson., Ser. A 115 (2) (1995) 260-264.

[69] Alexej Jerschow, Norbert Müller, 3D diffusion-ordered TOCSY for slowly diffusing molecules, J. Magn. Reson., Ser. A 123 (2) (1996) 222-225.

[70] Alexej Jerschow, Norbert Müller, Suppression of Convection Artifacts in Stimulated-Echo Diffusion Experiments. Double-Stimulated-Echo Experiments, J. Magn. Reson. 125 (2) (1997) 372-375.

[71] Xiaoai Guo, Esther Laryea, Manfred Wilhelm, Burkhard Luy, Hermann Nirschl, Gisela Guthausen, Diffusion in Polymer Solutions: Molecular Weight Distribution by PFG-NMR and Relation to SEC, Macromol. Chem. Phys. 218 (1) (2017) 1600440, https://doi.org/10.1002/macp.201600440.

[72] Robert C. Hardy, Robert L. Cottington, Viscosity of deuterium oxide and water in the range 5 to 125 C, J. Res. Natl. Bur. Stand 42 (6) (1949) 573, https://doi. org/10.6028/jres.042.049.

[73] M.C. Carey, D.M. Small, Micelle Formation by Bile Salts: Physical-Chemical and Thermodynamic Considerations, Arch. Intern. Med. 130 (4) (1972) 506-527.

[74] K. Fontell, The Micellar Structure Of Bile SaltSolutions: Laboratory for Surface Chemistry ("Ytkemiska Laboratoriet") at the Royal Swedish Academy of Engineering Sciences, surface chemistry, Stockholm, Sweden, Elsevier 1965, pp. 252-267. 
[75] L.B. Pártay, M. Sega, P. Jedlovszky, Morphology of Bile Salt Micelles as Studied by Computer Simulation Methods, Langmuir 23 (24) (2007) 12322-12328.

[76] N. Pandit, T. Trygstad, S. Croy, M. Bohorquez, C. Koch, Effect of Salts on the Micellization, Clouding, and Solubilization Behavior of Pluronic F127 Solutions, J. Colloid Interface Sci. 222 (2) (2000) 213-220.

[77] A.J. Clulow, A. Parrow, A. Hawley, J. Khan, A.C. Pham, P. Larsson, C.A.S. Bergström, B.J. Boyd, Characterization of Solubilizing Nanoaggregates Present in Different Versions of Simulated Intestinal Fluid, J. Phys. Chem. B 121 (48) (2017) 10869-10881.

[78] A. Parrow, P. Larsson, P. Augustijns, C.A.S. Bergström, Molecular Dynamics Simulations on Interindividual Variability of Intestinal Fluids: Impact on Drug Solubilization, Mol. Pharmaceutics 17 (10) (2020) 3837-3844.

[79] S. Boros, Z. Gáspári, G. Batta, Chapter One - Accurate NMR Determinations of Proton-Proton Distances, Annu. Rep. NMR Spectrosc. 94 (2018) 1-39. 